\title{
Article Title
}

An acylsugar-deficient Nicotiana benthamiana strain for aphid and whitefly research

\section{Running title}

Acylsugars protect Nicotiana benthamiana

\section{Author names}

Honglin Feng ${ }^{\mathrm{a}}$, Lucia Acosta-Gamboa ${ }^{\mathrm{b}}$, Lars H. Kruse, ${ }^{\mathrm{c}, \mathrm{f}}$, Jake D. Tracy ${ }^{\mathrm{d}}$, Seung Ho Chung ${ }^{\mathrm{a}}$, Alba Ruth Nava Fereira ${ }^{\mathrm{e}}$, Sara Shakir ${ }^{\mathrm{a}, \mathrm{g}}$, Hongxing Xu $\mathrm{Xu}^{\mathrm{a}, \mathrm{h}}$, Garry Sunter ${ }^{\mathrm{e}}$, Michael A. Gore ${ }^{\mathrm{b}}$, Clare L. Casteel ${ }^{\mathrm{d}}$, Gaurav D. Moghe ${ }^{\mathrm{c}}$, Georg Jander ${ }^{\mathrm{a}}$

\section{Author Affiliations}

${ }^{a}$ Boyce Thompson Institute, Ithaca NY, USA

$16{ }^{\mathrm{b}}$ Plant Breeding and Genetics Section, School of Integrative Plant Science, Cornell University,

17 Ithaca NY, 14853, USA

'Plant Biology Section, School of Integrative Plant Science, Cornell University, Ithaca NY, 14853, USA

${ }^{\mathrm{d}}$ Plant-Microbe Biology and Plant Pathology Section, School of Integrative Plant Science,

21 Cornell University, Ithaca NY, 14853, USA

22 'Department of Biology, University of Texas San Antonio, San Antonio TX, 78249, USA

23 'Present address: Michael Smith Laboratories, University of British Columbia, Vancouver, BC,

24 V6T 1Z4, Canada

25 'Present address: Gembloux Agro-Bio Tech Institute, the University of Liege, Gembloux,

26 Belgium

${ }^{\mathrm{h}}$ Present address: College of Life Science, the Shaanxi Normal University, Xi' an, China

\section{*Correspondence:}

32 Georg Jander

33 Boyce Thompson Institute

34 Ithaca, NY 14853

35 USA

36 Phone: 607-254-1365

37 Email: gj32@cornell.edu 


\section{Abstract}

39 Nicotiana benthamiana is used extensively as a platform for transient gene expression and as a

40 model system for studying plant-virus interactions. However, many tobacco-feeding generalist

41 herbivores, including Myzus persicae (green peach aphid), Bemisia tabaci (whitefly),

42 Macrosiphum euphorbiae (potato aphid), Heliothis virescens (tobacco budworm), Trichoplusia

$43 n i$ (cabbage looper), and Helicoverpa zea (corn earworm), grow poorly on N. benthamiana,

44 limiting its utility for research on plant-insect interactions. Using CRISPR/Cas9, we generated

45 knockout mutations in two $N$. benthamiana acylsugar acyltransferases, ASAT1 and ASAT2, which

46 contribute to the biosynthesis of insect-deterrent acylsucroses. Whereas asat1 mutations reduced

47 the abundance of two predominant acylsucroses, asat 2 mutations caused almost complete

48 depletion of foliar acylsucroses. The tested hemipteran and lepidopteran species survived, gained

49 weight, and/or reproduced significantly better on asat 2 mutant plants than on wildtype $N$.

50 benthamiana. Furthermore, both asat1 and asat 2 mutations reduced the water content and

51 increased the temperature of leaves, indicating that foliar acylsucroses can protect against

52 desiccation. Two experiments demonstrated the utility of the $N$. benthamiana asat 2 mutant line

53 for insect bioassays. Transmission of turnip mosaic virus by $M$. persicae was significantly

54 improved by an asat 2 mutation. Tobacco rattle virus constructs were used for virus-induced gene

55 silencing of acetylcholinesterase, squalene synthase, toll-like receptor 7 , and tubulin-specific

56 chaperon $D$ genes in $B$. tabaci, an experiment that would have been difficult with wild-type $N$.

57 benthamiana due to high insect mortality. Additionally, the absence of acylsugars in asat 2

58 mutant lines will simplify transient expression assays for the functional analysis of acylsugar

59 biosynthesis genes from other Solanaceae. 


\section{Introduction}

63 Nicotiana benthamiana, a wild tobacco species that is native to Australia, is commonly used by

64 plant molecular biologists as model system for laboratory research. Susceptibility to a wide

65 variety of plant viruses has made $N$. benthamiana a popular model for fundamental studies of

66 plant-virus interactions (Goodin et al., 2008). Scientists have developed N. benthamiana as a

67 transgene expression powerhouse by engineering viral vectors to express heterologous genes,

68 including fluorescent reporter genes to visualize cell structures (Bally et al., 2018). Antibodies,

69 biofuel compounds, and other protein and metabolite products have been produced in $N$.

70 benthamiana (Arntzen, 2015; Powell, 2015). Virus-induced gene silencing (VIGS), which is

71 employed to study gene function in a variety of plant species, was originally developed in $N$.

72 benthamiana (Velásquez et al., 2009; Hayward et al., 2011). Recently, high-efficiency

73 CRISPR/Cas9 gene editing of germline cells using virus-encoded guide RNA (gRNA) was

74 demonstrated for the first time in N. benthamiana (Ellison et al., 2020). Although $N$.

75 benthamiana is hyper-susceptible to many plant viruses, it is not a good host for three virus-

76 transmitting Hemiptera, Myzus persicae (green peach aphid; Thurston, 1961; Hagimori et al.,

77 1993), Bemisia tabaci (whitefly; Simon et al., 2003), and Macrosiphum euphorbiae (potato

78 aphid) that otherwise grow well on cultivated tobacco (Nicotiana tabacum).

The poor growth of generalist insect herbivores on $N$. benthamiana may be attributed in part to glandular trichomes. These epidermal secretory structures on the leaf surface of $\sim 30 \%$ of vascular plants (Weinhold and Baldwin, 2011; Glas et al., 2012) have been found to play a crucial defensive role in several ways: as a physical obstacle for insect movement on the plant surface (Cardoso, 2008), entrapment (Simmons et al., 2004), synthesis of volatiles and other defensive metabolites (Laue et al., 2000; Schilmiller et al., 2010; Glas et al., 2012), and production of proteins that repel herbivores (phylloplane proteins, e.g. T-phylloplanin; Shepherd and Wagner, 2007). In addition to their defensive functions, glandular trichomes also protect

87 plants from abiotic stresses such as transpiration water loss and UV irradiation (Karabourniotis et 88 al., 1995).

There are two main types of glandular trichomes on $N$. benthamiana leaves, large swollen-stalk trichomes and small trichomes that are capped by a secretory head with one, two,

91 or four cells (Slocombe et al., 2008). The large trichomes have been shown to secrete

92 phylloplane proteins in N. tabacum. The small trichomes, which are the most abundant on 
93 tobacco leaf surfaces, secrete exudates, including acylsugars (Wagner et al., 2004; Slocombe et

94 al., 2008). Detached trichomes, a mixture of the large and small trichomes, from $N$. benthamiana

95 are able to synthesize acylsugars (Kroumova and Wagner, 2003), and the secretory head cells

96 alone are able to synthesize acylsugars in N. tabacum (Kandra and Wagner, 1988).

97 Acylsugars, generally sucrose or glucose esterified with aliphatic acids of different chain

98 lengths (Figure 1), are abundant insect-deterrent metabolites produced by Solanaceae glandular

99 trichomes (Arrendale et al., 1990; Slocombe et al., 2008; Moghe et al., 2017). Specific

100 acylsugars are associated with aphid-resistant Nicotiana species, while not being detected in

101 more susceptible species in this genus (Hagimori et al., 1993). Furthermore, relative to cultivated

102 tomatoes (Solanum lycopersicum), acylsugars provide wild tomatoes (Solanum pennellii) greater

103 resistance against $M$. persicae (Rodriguez et al., 1993). Diacylsucrose protects crops against

104 tobacco aphids (Myzus persicae nicotianae), B. tabaci, and two-spotted spider mites

105 (Tetranychus urticae) (Chortyk et al., 1996; Alba et al., 2009). The synthetic sucrose octanoate

106 (an analog of Nicotiana gossei sugar esters) is effective in the field against Asian citrus psyllids

107 (Diaphorina citri), citrus leafminer (Phyllocnistis citrella), and a mite complex (including Texas

108 citrus mite, red spider mite, and rust mite) (McKenzie and Puterka, 2004; McKenzie et al.,

109 2005). Interestingly, acylsucroses in Nicotiana attenuata are metabolized to volatile fatty acids

110 by neonate Manduca sexta (tobacco hornworm) larvae, thereby tagging these larvae and

111 attracting predatory ants, Pogonomyrmex rugosus (Weinhold and Baldwin, 2011).

112 Acylsugars and leaf surface lipids more generally may contribute to plant drought

113 tolerance. Transcriptomic studies of drought-tolerant S. pennellii populations showed that lipid

114 metabolism genes are among those that are most responsive to drought stress (Gong et al., 2010;

115 Egea et al., 2018). Additionally, the abundance of acylsugars on the leaf surface in a native $S$.

116 pennellii population was associated with drought tolerance (Fobes et al., 1985). Acylsugars also

117 are reported to provide protection against drought stress conditions in Solanum chilense (O'

118 Connell et al., 2007). Similarly, abundant accumulation of acylsugars with C7-C8 acyl groups in

119 the desert tobacco (Nicotiana obtusifolia) was suggested to provide this species with high

120 drought tolerance for its desert environment (Kroumova et al., 2016). Although the mechanism is

121 not completely understood, it has been proposed that the polar lipids reduce the surface tension

122 of adsorbed dew water, thereby allowing the leaves absorb more condensed water on the surface

123 (Fobes et al., 1985). 
More recently, enzymes involved in the biosynthesis of acylsugars have been identified.

125 Four acylsugar acyltransferases (ASATs), SlASAT1, SlASAT2, SlASAT3, and SlASAT4, have

126 been biochemically characterized in cultivated tomato (Fan et al., 2016). SlASAT1 catalyzes the

127 first step of sucrose acylation, using sucrose and acyl-CoA to generate monoacylsucroses via

128 pyranose $\mathrm{R}_{4}$ acylation (Fan et al., 2016). SlASAT2 uses the product of SlASAT1 (e.g.

$129 \mathrm{~S} 1: 5\left(\mathrm{iC}^{\mathrm{R} 4}\right)$ ) and acyl-CoA donor substrates (e.g. iC4-CoA, aiC5-CoA, nC10-CoA, and nC12-

$130 \mathrm{CoA}$ ) to generate diacylsucroses (Fan et al., 2016). Further, SlASAT3 uses the diacylsucroses

131 generated by SIASAT2 to make triacylsucroses by acylating the diacylsucrose five-membered

132 (furanose) ring (Fan et al., 2016). Then, SlASAT4 makes tetraacylsucroses by acetylating

133 triacylsucroes using C2-CoA (Schilmiller et al., 2012). Notably, SlASAT4 (formerly SlASAT2)

134 is specifically expressed in the trichomes, where acylsugar acetylation occurs (Schilmiller et al.,

135 2012). The expression and activity of ASATs varies among different plant species (including the

136 order of the ASAT reactions in the pathway), which likely contributes to the observed trichome

137 chemical diversity (Kim et al., 2012). Although ASATs have been most intensively studied in

138 tomato, ASAT genes also have been annotated in the available Nicotiana genomes (Gaquerel et

139 al., 2013; Van et al., 2017; Egan et al., 2019). However, ASAT genes in N. benthamiana have not

140 been annotated and characterized previously, and their functions in protection against insect

141 pests and desiccation remained unknown.

142 The goal of this study was to confirm the role of acylsugars in N. benthamiana resistance

143 to insect feeding, as well as to create an insect-susceptible ASAT mutant line to facilitate use of

$144 N$. benthamiana for laboratory research on plant-insect interactions. We identified two ASAT

145 genes in N. benthamiana, NbASAT1 and NbASAT2. Using CRISPR/Cas9 to create mutant lines,

146 we showed that knockout of both $N b A S A T 1$ and $N b A S A T 2$ reduced acylsugar content. NbASAT2

147 mutations allowed increased survival, growth, and/or reproduction of six tested insect species.

148 Additionally, decreased water content and elevated leaf temperature in NbASAT1 and NbASAT2

149 mutants indicated that $N$. benthamiana acylsugars contribute to protection against desiccation.

$151 \quad$ Results

152 Identification of ASAT1 and ASAT2 in N. benthamiana

153 Using reciprocal comparisons to confirmed Solanaceae ASAT genes (Moghe et al., 2017), we 154 identified three highly homologous sequences in the $N$. benthamiana genome: 
156 (Bombarely et al., 2012) (gene identifiers are from annotations at solgenomics.net). Whereas

157 Niben101Scf02239Ctg025 and Niben101Scf22800Ctg001 were annotated as a full-length coding

158 sequences with strong coverage in available RNAseq datasets, Niben101Scf14179Ctg028 was

159 annotated as a pseudogene because it appears to be a fragment of the predicted cDNA

160 Niben101Scf141790g02010.1, but with no coverage in available RNAseq datasets. The

161 Niben101Scf02239Ctg025 and Niben101Scf22800Ctg001 sequences were confirmed in a more

162 recently assembled $N$. benthamiana genome (Schiavinato et al., 2019). In this assembly, the

163 pseudogene Niben101Scf14179Ctg028 was annotated as part of Niben101Scf02239Ctg025, and

164 there were no additional annotated ASAT candidates.

165 To infer ASAT evolution and function, we constructed a protein phylogenetic tree of 166 previously annotated Solanaceae ASATs (Figures 2, S1 and S2; Tables S1 and S2). In this tree,

167 Niben101Scf02239Ctg025 formed a monophyletic group with other ASATs, including the

168 biochemically characterized $S s$ ASAT1, PaASAT1 and HnASAT1. Therefore, we named

169 Niben101Scf02239Ctg025 as $N$. benthamiana ASAT1 (NbASAT1). Niben101Scf22800Ctg001

170 formed a monophyletic group with other ASATs including the biochemically characterized

171 NaASAT2, HnASAT2, and PaASAT2. Therefore, we named Niben101Scf22800Ctg001 as $N$.

172 benthamiana ASAT2 (NbASAT2). Notably, the ASAT2 monophyletic group also included the

173 biochemically characterized SpASAT1, SlASAT1, and SnASAT1 (Figure 2).

174

\section{Generation of ASAT mutants}

176 Using CRISPR/Cas9 coupled with tissue culture, we obtained two independent homozygous

177 mutants for both NbASAT1 and NbASAT2. asat1-1 has a five-nucleotide deletion at the gRNA3

178 cutting site and a single nucleotide insertion at the gRNA2 cutting site, leading to a frameshift

179 between gRNA3 and gRNA2. asat1-2 has a 318-nucleotide deletion between the gRNA3 and

180 gRNA2 cutting sites (Figure 3A). asat2-1 has a single-nucleotide deletion at the gRNA3 cutting

181 site and single-nucleotide insertion at the gRNA2 cutting site, leading to a translation frame shift

182 between the two sites. asat2-2 has a 115-nucleotide deletion at the gRNA3 cutting site and a

183 single-nucleotide insertion at the gRNA2 cutting site (Figure 3B). Homozygous mutants were

184 confirmed by DNA sequencing in the T2 generation, and this generation was used for all

185 experiments. 


\section{ASAT2 knockout depletes acylsugar biosynthesis}

188 We quantified the acylsugar content in the ASAT mutants by LC/MS, comparing to that of wildtype $N$. benthamiana plants. In the LC/MS profile of $N$. benthamiana leaf surface washes, we characterized twelve mass features as acylsucroses based on their characteristic peaks and neutral losses (Figure S3A). In negative electron spray ionization mode, the characteristic peak

192 features included the mass of 341.11 for sucrose, 509.22 for sucrose $+\mathrm{C} 2+\mathrm{C} 8,467.21$ for

193 sucrose + C8, 495.21 for sucrose + C7, and 383.12 for sucrose + C2; the neutral loss peaks

194 included mass for 126.10 for $\mathrm{C} 8$ (acyl-chain with 8 carbons), 129.09 for $\mathrm{C} 7+\mathrm{H}_{2} \mathrm{O}$, and 59.01 for

$195 \mathrm{C} 2+\mathrm{H}_{2} \mathrm{O}$ (Figure S3B). Those twelve $\mathrm{m} / \mathrm{z}$ ratios included 383.12, 467. 21, 509.22, 555.23,

$196593.32,621.31$, M625.31, 635.32, 639.32, 667.32, 671.30, 681.34 (Figure S3A). Based on their

197 MS/MS peak features, retention times and relative abundances, we predicted that the identified

198 mass features are mainly derived from two acylsucroses as formate or chloride adducts, pathway

199 intermediates, and/or resulted from in-source fragmentation. We named the two acylsucroses

200 S3:17(2,7,8) and S3:18(2,8,8) (in the nomenclature, "S" refers to the sucrose backbone, "3:18"

201 indicates three acyl chains with total eighteen carbons, and the length of each acyl chain is

202 shown in parentheses) (Figure 1; Figure S3A).

In wildtype plants, $\mathrm{S} 3: 18(2,8,8)$ is the dominant acylsucrose, whereas $\mathrm{S} 3: 17(2,7,8)$ has

204 relatively low abundance (Figure 4). S2:16(8,8) and S2:15(7,8), which may be biosynthetic

205 pathway intermediates for S3:18(2,8,8) and S3:17(2,7,8), respectively, are present at lower levels

206 (Figure 4). Compared to wildtype N. benthamiana, both asat2-1 and asat2-2 were almost

207 completely depleted in both S3:17(2,7,8) and S3:18(2,8,8), as well as in the two predicted

208 biosynthetic intermediates S2:15(7,8) and S2:16(8,8) (Figure 4). For asat1-1 and asat1-2, the

209 detected acylsucroses were less abundant, and significantly reduced in asat1-1 (Figure 4).

210 Although acylsugar content was significantly reduced in the ASAT mutants, the structure and

211 abundance of trichomes on the leaf surface were not visibly changed (Figure S4).

213 Insect performance is improved on ASAT2 mutant lines

214 We used asat1 and asat2 mutant lines to test the role of acylsugars in protecting $N$. benthamiana

215 against insect pests. After placing synchronized first-instar aphids on mutant and wildtype $N$.

216 benthamiana leaves, we monitored survival and growth over time. Significant improvements in 
217 aphid survivorship were observed as early as at 2 days post-feeding $(p<0.001)$ on the asat2-1

218 and asat2-2 mutants and increased until the end of the 5-day monitoring period $(p<0.001)$

219 (Figure 5A and Table S3). After 5 days of feeding, surviving aphids on both asat1 and asat2

220 plants were larger than those on wildtype plants (Figures 5B). When we measured progeny

221 production by five adult aphids over a period of seven days, an average of more than 200

222 nymphs were produced on the asat 2 mutants, significantly more than the number of nymphs

223 produced on either wildtype or asat1 mutants ( $p<0.05$, Figure 5C). In aphid choice assays with

224 detached leaves, they preferentially chose asat2-1 mutant leaves relative to wildtype and asat1-1

225 leaves $(p<0.05$, Figures 5D-F). A preference for asat2-1 and asat2-2 leaves was consistently

226 observed in choice assays involving any pairwise combination with wildtype, asat1-1, and asat1-

2272 leaves ( $p<0.001$, Chi-square test, Figures 5D-F and S5). No M. persicae preference was

228 observed when comparing wildtype $N$. benthamiana and asat1 mutants ( $p>0.05$, Figures 5D-F

229 and S5).

230 When aphid colonies were allowed to grow long-term on asat2-1 mutant and wildtype $N$.

231 benthamiana in the same growth chamber, there were many more aphids on the mutant plants

232 (Figure S6A,B), likely resulting from a combination of host plant choice and increase growth on

233 the asat2-1 mutant. It is noteworthy that, on the asat2-1 mutant plants, aphids were feeding on

234 the more nutritious younger leaves, which tend to be better-defended in plants. By contrast, on

235 wildtype $N$. benthamiana aphids were only were able to feed on older, senescing leaves and were

236 primarily on the abaxial surface. Consistent with the increased aphid presence, growth of the

237 asat2-1 mutant plants was visibly reduced relative to wildtype $N$. benthamiana (Figure S6B).

238 Given the almost identical phenotypes of asat2-1 and asat2-2 mutants, subsequent insect assays

239 were conducted with T2 progeny of the asat2-1 line, which also were confirmed by PCR to

240 contain the Cas9 transgene.

241 As we observed with $M$. persicae, the asat2-1 mutation improved the M. euphorbiae

242 performance on $N$. benthamiana (Figure 6A-C). Significantly increased M. euphorbiae survival

243 was observed after 24 hours on asat2-1 compared to wildtype ( $p<0.001$, Figure 6A).

244 Additionally, significantly more nymphs were produced by adult $M$. euphorbiae in the course of

24524 hours on asat $2-1$ than on wildtype ( $p<0.05$, Figure 6B). In choice assays, potato aphids

246 preferentially chose the asat $2-1$ leaves over wildtype leaves ( $p<0.001$, Figure 6C). Whereas we 
were not able to establish an $M$. euphorbiae colony on wildtype $N$. benthamiana, the aphids readily formed colonies on the asat2-1 mutant plants (Figure 6D).

Survival of B. tabaci was greatly increased on the asat $2-1$ mutant line relative to wildtype

(Figure 6E). Moreover, whiteflies laid significantly fewer eggs on wildtype $N$. benthamiana than on asat2-1 mutant plants over three days (Figure 6F). Dead adult whiteflies were observed on wildtype plants (Figure S7A), and it was not possible to establish a reproducing colony. By contrast, after 23 days of feeding on $N$. benthamiana, whiteflies of different life stages were

254 observed on asat2-1 mutant plants (Figure S7B-D). In choice assays with mutant and wildtype

255 plants in the same cage, $B$. tabaci preferentially settled on asat2-1 plants (72\%) over wildtype

256 (26\%) in a 24-hour experiment (Figure 6G). Notably, all whiteflies on asat2-1 were alive after

25724 hours, whereas about half of the whiteflies found on the wildtype plants were dead at the 258 same time point (Figure 6F).

259 To determine whether depletion of acylsugars in ASAT2 mutants improves the

260 performance of lepidopteran herbivores on $N$. benthamiana, we conducted experiments with 261 Helicoverpa zea (corn earworm), Heliothis virescens (tobacco budworm) and Trichoplusia ni

262 (cabbage looper). When neonates were placed on the leaves of wildtype or asat2-1 mutant $N$.

263 benthamiana, no H. zea caterpillars were recovered (Figure 7A). Survivorship of H. virescens

264 and T. ni larvae on N. benthamiana was low, and the mass of the surviving larvae after ten days

265 was not significantly increased on the mutant line relative to wildtype (Figure 7B,C). Due to the

266 low survival of neonates, we repeated the caterpillar bioassay using five-day-old larvae that had

267 been reared on artificial diet. Almost all H. zea and H. virescens larvae survived for seven days

268 on wildtype and asat2-1 mutant plants, and survival of $T$. ni caterpillars was higher on asat2-1

269 than on wildtype plants (Figure 7D-F). The relative growth rates of surviving H. zea, H.

270 virescens, and T. ni larvae were higher on the asat $2-1$ mutant by $35 \%, 47 \%$, and 99\%,

271 respectively, than on wildtype plants (Figure 7D-F).

273 Virus transmission by aphids is increased on asat2-1 mutant plants

274 To demonstrate the use of asat2-1 mutants for aphid experiments, we measured M. persicae

275 transmission of a GFP-expressing turnip mosaic virus (TuMV-GFP; Lellis et al., 2002; Casteel et

276 al., 2015) using all combinations of wildtype and asat2-1 plants as virus donors and recipients,

277 respectively (Figure 8). The earliest GFP signals in the recipient plants were visible under UV 
light on day 4 after TuMV-GFP transmission. Successful transmission and virus replication was monitored until day 9, when no plants developed additional visible TuMV-GFP infections relative to the previous day (Figure 8 ). The asat2-1 $\rightarrow$ asat $2-1$ transmission group showed the highest final TuMV-GFP infection rate (73\%), significantly higher than that observed in the other three groups $(p<0.05$, ANOVA). Relative to the wildtype $\rightarrow$ wildtype TuMV-GFP transmission, transmission was not significantly increased in the asat2-1 $\rightarrow$ wildtype and wildtype $\rightarrow$ asat2-1 groups. The significantly higher virus transmission rate by aphids in the asat2-1 $\rightarrow$ asat2-1 group is consistent with the feeding preference of $M$. persicae for asat2-1 mutant plants (Figure 5).

\section{The asat2-1 mutant is suitable for plant-mediated VIGS in whiteflies}

289 To demonstrate the utility of the asat2-1 mutant for whitefly experiments, we performed plantmediated virus-induced gene silencing (VIGS) using tobacco rattle virus (TRV; Hayward et al., 2011). We selected two previously validated B. tabaci RNAi targets, AchE (acetylcholinesterase;

292 Malik et al., 2016) and TLR7 (toll-like receptor 7; Chen et al., 2015), as well as two predicted

293 horizontally transferred genes (Chen et al., 2016), SQS (squalene synthase) and TSCD (tubulin-

294 specific chaperone $D$ ), that had not been previously investigated in whiteflies. When whiteflies

295 were place on $N$. benthamiana infected with TRV VIGS constructs, expression of all four target

296 genes was significantly reduced after one day of feeding (Figure 9A). After seven days of

297 feeding, the expression of $A c h E$ and $S Q S$ was significantly reduced relative to whiteflies on

298 empty vector control plants, but expression of LTR7 and TCSD was not (Figure 9B). After seven

299 days, survivorship of the whiteflies on VIGS plants was significantly reduced relative to control

300 plants with empty vector or GFP control TRV infections (Figure 9C). Consistent with the low

301 survival observed in previous experiments (Figure 6E), whitefly survival on TRV-infected

302 wildtype $N$. benthamiana was very low (Figure 9D), never exceeding 10\%. None of the VIGS

303 constructs decreased survival to a lower level than on the empty vector and TRV-GFP control

304 plants.

307 While conducting aphid choice assays with detached leaves (Figures 5D-F and 6C), we noticed 308 that the mutant leaves dried out faster than wildtype leaves. This effect was quantified using 
309 detached-leaf assays, in which asat2 leaves lost significantly more water over 24 hours than

310 leaves from either wildtype or asat1 leaves (Figures 10A and S8A). When plants are subjected to

311 drought, high/low temperature, salinity, herbivores, or other stresses, they absorb and reflect

312 specific wavelengths of light, which have been used to determine vegetation indices. For

313 example, the water band index (WBI) (Penuelas et al., 1993) has been used to monitor changes

314 in the plant canopy/leaf water content. Using hyperspectral imaging, we determined that the leaf

315 water content of intact plants, as measured by the WBI, was significantly lower in asat2 mutants

316 than in wildtype (Figures 10B and S8B). Although the asat1 mutants did not lose water faster

317 than wildtype in detached leaf assays (Figure 10A), the leaf water content in asat1 mutants was

318 significantly lower than wildtype (Figures 10B and S10B). Measurement of leaf temperature by

319 thermal imaging showed that, consistent with the reduced leaf water content, the leaf temperature

320 of the acylsugar mutants was significantly higher than that of wildtype plants (Figures 10C and

321 S10C).

\section{Discussion}

324 We were able to identify only two ASAT genes, $N b A S A T 1$ and NbASAT2, as well as a fragmented 325 pseudogene, in the $N$. benthamiana genome (Table S2). By contrast, in other Nicotiana species, 326 there are larger numbers of predicted ASATs, e.g. one ASAT1, one ASAT2, and 20 ASAT3-like

327 genes in N. attenuata (Gaquerel et al., 2013; Van et al., 2017), 35 ASAT3-like genes in N.

328 tabacum, and 19 ASAT3-like genes in N. tomentosiformis (Egan et al., 2019). Given the

329 relatively small number of predicted $A S A T$ genes in $N$. benthamiana, other enzymes may also be

330 involved in acylsugar biosynthesis. In the Solanaceae, two common pathways are known for

331 aliphatic acid elongation via acetate: the fatty acid synthase (FAS) and the alpha-ketoacid

332 elongation ( $\alpha \mathrm{KAE}$ ) pathways (Kroumova and Wagner, 2003). In the FAS pathway, two carbons

333 from an acetyl-acyl carrier are retained per elongation cycle, whereas in the $\alpha \mathrm{KAE}$ pathway, one

334 carbon is retained per elongation cycle (Kroumova and Wagner, 2003). Knockdown of E1- $\beta$

335 branched-chain $\alpha$-keto acid dehydrogenase (BCKD) significantly reduces acylsugars in $N$.

336 benthamiana (Slocombe et al., 2008). Additionally, Isopropylmalate Synthase 3 (IPMS3) in

337 cultivated and wild tomatoes (Ning et al., 2015) and Acyl-Sucrose Fructo-Furanosidase 1

338 (ASFF1) in wild tomato (Leong et al., 2019) are involved in determining acylsugar composition. 
339 Further studies will be needed to characterize other genes involved in the N. benthamiana 340 acylsugar biosynthesis pathway.

341 Acylsugars can be categorized as sucrose or glucose esters based on the sugar cores,

342 which are decorated with varying numbers or lengths of acyl chains (Kim et al., 2012). Whereas

343 some wild tomatoes produce a mixture of acylsucroses and acylglucoses, we observed only

344 acylsucroses (Figures 1 and 4), consistent with previous identification of these compounds in $N$.

345 benthamiana (Matsuzaki et al., 1989; Matsuzaki et al., 1992; Hagimori et al., 1993; Slocombe et

346 al., 2008). Nevertheless, it has been reported that $N$. benthamiana produces acylglucoses,

347 although in lower abundance than acylsucroses (Hagimori et al., 1993), and one glucose ester

348 structure has been proposed (Matsuzaki et al., 1992). Our failure to detect acylglucoses may be

349 explained by the use of different isolates of $N$. benthamiana, growth conditions, growth stage of

350 plants, and/or the detection methods. Whereas we used 1-month-old plants and LC/MS for our

351 assays, Matsuzaki et al. (1992) used 3-month-old plants and GC/MS to detect acylglucoses in

352 N. benthamiana.

353 Abundance of the characterized acylsugars was reduced to a greater extent in $N$.

354 benthamiana asat2 than in asat1 mutants (Figure 4). The smaller reduction of acylsugars in

355 asat1 mutants suggests that either $\mathrm{NbASAT} 2$ functions upstream of NbASAT1 in the acylsugar

356 biosynthesis pathway, but partially complements NbASAT1 activity; or NbASAT1 and

357 NbASAT2 have similar functions in the biochemical pathway, but NbASAT2 had higher

358 abundance or enzymatic activity. It is not known whether $N$. benthamiana ASATs are

359 monomeric or multimeric, but BAHD acyltransferases generally are monomeric enzymes

360 (D'Auria, 2006), suggesting that heterodimers between ASAT1 and ASAT2 are unlikely to affect

361 the observed phenotypes.

362 In the ASAT phylogenetic tree (Figure 2), NbASAT2 is closely related to some

363 biochemically characterized ASAT1 proteins in other Solanaceae species, including the

364 SpASAT1, SlASAT1, and SnASAT1. Those ASAT1s have some substrate overlap with the

365 ASAT2s found in the corresponding species, indicating that ASAT2 has moved toward utilizing

366 the ASAT1 substrate in these species over time (Moghe et al., 2017). The final activity shift that

367 has become fixed in the Solanum genus, most likely occurred after the divergence of the

368 Solanum and Capsicum clades (Moghe et al., 2017). However, if our hypothesis of partial

369 complementation of $N b$ ASAT1 by $N b$ ASAT2 is correct, it may flag a transition stage or suggest 
independent Nicotiana-specific evolution of the ASAT1 and ASAT2 functions. Based on previous knowledge of BAHD activities (Moghe et al., 2017), we postulate that $S 2: 15(7,8)$ and S2:16 $(8,8)$ are produced by NbASAT1 and NbASAT2, whereas the acetylation is carried out by another unrelated BAHD enzyme - not unlike the distantly related SlASAT4 and Salpiglossis sinuata ASAT5 enzymes (Schilmiller et al., 2015; Moghe et al., 2017). Further characterization will be required to identify specific acyltransferase enzyme activities in $N$. benthamiana. consistent with previous reports from other Solanaceae (Fobes et al., 1985; O' Connell et al., 2007; Kroumova et al., 2016) and is likely an adaptation to the seasonally arid native habitat of N. benthamiana in northwestern Australia (Goodin et al., 2008; The Australasian Virtual

380 Herbarium, https://avh.ala.org.au). Relative to asat1-1 and asat1-2 mutants, the lower acylsugar content of asat2-1 and asat2-2 mutants (Figure 4), resulted in more rapid water loss in detached

382 leaves (Figures 10A and S8A). However, despite the only partial decrease in the acylsugar

383 content of asat1 mutants, the decreases in water content and increases in leaf temperature of

384 intact plants were similar to those of asat2 mutants (Figures 10B,C and S8B,C).

Acylsugars with $\mathrm{C}_{7-12}$ chains have been shown to be the most toxic sugar esters for small phloem-feeding Hemiptera such as aphids, Asian citrus psyllids, and whiteflies (Chortyk et al., 1996; McKenzie and Puterka, 2004; Song et al., 2006). Synthetic acylsucroses with di-heptanoic acid (C7), di-octanoic acid (C8), and di-nonanoic acid (C9) acyl groups showed the highest mortality in bioassays with M. persicae and B. tabaci (Chortyk et al., 1996; McKenzie and Puterka, 2004; Song et al., 2006). Nicotiana gossei, a tobacco species that produces mainly C7C8 acyl group acylsugars, has a high level of insect resistance relative to close relatives with acylsugar profiles that are not dominated by those with C7-C12 acyl groups (Thurston, 1961; Kroumova and Wagner, 2003). In N. benthamiana, the two most abundant acylsugars that we found contain $\mathrm{C} 7$ and predominantly $\mathrm{C} 8$ acyl groups, which is consistent with previous findings of mainly 5- and 6-methyl heptanoate (C8) in N. benthamiana (Kroumova and Wagner, 2003; Slocombe et al., 2008) and N. alata (Moghe et al., 2017). The almost complete depletion of acylsugars in our asat 2 mutants improved both hemipteran and lepidopteran performance, suggesting that the identified C8-chain acyl group acylsugars are providing insect resistance for N. benthamiana. 
We cannot rule out the possibility secondary effects that might also influence insect performance on acylsugar-depleted $N$. benthamiana. Specialized metabolites in other plants, for instance glucosinolates in Arabidopsis thaliana (Clay et al., 2009) and benzoxazinoids in Zea mays (Meihls et al., 2013), regulate callose deposition as a secondary defense response. It is not known whether acylsugars contribute to the regulation of other defense responses in $N$. benthamiana. The observation of numerous dead whiteflies on wildtype $N$. benthamiana plants in choice assays (Figure 6G), despite the option of moving to presumably more desirable asat2-1 mutant plants in the same cage, suggests that the acylsugars stickiness also plays a role in plant defense by immobilizing the insects. Both altered leaf turgor and leaf temperature (Figure $10 \mathrm{~B}, \mathrm{C})$ could affect insect feeding behavior and growth rate, though the specific effects on the six tested insect species cannot be determined without further research.

412 and VIGS of $M$. persicae genes, and transient expression of genes from other species to

413 determine their effects on aphid growth and reproduction (Ramsey et al., 2007; Bos et al., 2010;

414 Pitino and Hogenhout, 2013; Casteel et al., 2014; Elzinga et al., 2014; Rodriguez et al., 2014;

415 Krenz et al., 2015; Tzin et al., 2015; Mulot et al., 2016; Mathers et al., 2017; Del Toro et al,

416 2018; Cui et al., 2019; Worrall et al., 2019). However, the poor growth of many M. persicae

417 isolates on N. benthamiana (Thurston, 1961; Hagimori et al., 1993; Figure S6) hampers

418 experiments of this kind Similarly, due to the very poor survival of $B$. tabaci on wildtype plants

419 (Simon et al., 2003; Figure 6E), N. benthamiana has not been useful as a host plant for whitefly 420 research.

421 To demonstrate the utility of asat2 mutants as a tool for aphid research, we performed

422 TuMV-GFP transmission experiments using $M$. persicae. Our results show that asat2-1 plants 423 significantly increased virus transmission (Figure 8). Interestingly, efficient virus transmission 424 only occurred when both the virus donor and the recipient plants had the asat2-1 genotype. This 425 suggests that aphid behavior on asat2-1 plants, perhaps faster or more continuous probing, 426 promotes both uptake and delivery of TuMV. The higher virus transmission on asat2-1 mutant $427 N$. benthamiana will facilitate use of this model system for future research on plant-aphid-virus 428 interactions and factors that promote virus transmission.

429 The low survival of whiteflies on wildtype $N$. benthamiana (Figures 6E and 9D) makes it 430 difficult to assess the negative effects of gene expression silencing by TRV VIGS. By contrast, 
431 we were able to demonstrate reduced survival with all four tested whitefly genes with TRV

432 VIGS using asat2-1 mutant plants (Figure 9C). TLR7 and TSCD gene expression was reduced on

433 day 1 but not on day 7 in the VIGS experiment (Figure 10A,B), but nevertheless there was a

434 negative effect on whitefly survival over 7 days (Figure 10C). Two possible explanations for this

435 finding are: (i) There was a survivor bias in that we could only measure gene expression levels in

436 surviving aphids, and perhaps all aphids with efficient expression silencing of TLR7 and TSCD

437 were dead after 7 days. (ii) There may be gene expression compensation at the whole-insect level

438 over time, but not in specific tissues that affect insect survival. Quantitative PCR of fractionated

439 whiteflies would be necessary to determine the time course of TLR7 and TSCD expression

440 silencing and whether VIGS primarily affects gene expression in specific tissue types.

441 In addition to confirming the importance of two B. tabaci genes, AchE and TLR7, that

442 have been targeted by RNAi (Malik et al., 2016; Chen et al., 2015), we chose two previously

443 uncharacterized horizontally transferred genes SQS and TSCD, as VIGS targets. Bemisia tabaci

444 MEAM1 has at least 142 horizontally transferred genes from bacteria and fungi (Chen et al.,

445 2016). Given that horizontal transfer of functionally expressed microbial genes into insect

446 germlines is rare on an evolutionary timescale, there is likely a selective advantage to having

447 these genes expressed in whiteflies. This was confirmed by the observation that VIGS of both

$448 S Q S$ and TSCD reduced whitefly survival relative to control plants (Figure 9C,D). Transient

449 expression knockdown of $S Q S, T S C D$, and other horizontally transferred genes will enable future

450 research to study the functions of these genes in whitefly metabolism. Due to their importance

451 for whitefly survival, as well their absence in beneficial insects such as ladybugs and lacewings,

452 horizontally transferred genes also are attractive targets for controlling whiteflies by RNA

453 interference. The established $N$. benthamiana VIGS system, which allows rapid cloning of

454 targets by Gateway recombination (Liu et al., 2002), will allow rapid screening of other

455 horizontally transferred genes to identify ones that would be most suitable for whitefly control on

456 crop plants by RNA interference.

457 Although H. zea, H. virescens, and T. ni larvae grow well on cultivated tobacco, neonate

458 larvae had a low survival rate on both wildtype asat2-1 N. benthamiana (Figure 7A-C). There

459 was a higher survival rate with five-day-old larvae of the three tested species, which all grew

460 significantly better on asat2-1 mutants than on wildtype $N$. benthamiana (Figure 7D-F). Thus, $N$.

461 benthamiana acylsugars likely provide at least some protection against lepidopteran pests. 
462 However, the high mortality of neonate larvae on asat2-1 plants suggests that either residual

463 acylsugars or as yet unknown resistance mechanisms in $N$. benthamiana can provide protection.

464 Additional mutations that decrease insect resistance, perhaps regulatory genes such as COII or

465 genes affecting the production of other specialized metabolites, will be necessary to facilitate $N$.

466 benthamiana experiments with H. zea, H. virescens, T. ni, and other commonly studied

467 lepidopteran species.

468 The Cas 9 transgene, which is still present in the asat2-1 mutant line that we used for

469 most of our experiments, may facilitate further mutagenesis. In a recently described method

470 (Ellison et al., 2020) Cas9-transgenic N. benthamiana plants were infected with TRV carrying

471 gRNAs linked to phloem movement sequences. Seeds harvested from these plants had a high

472 frequency of homozygous knockout mutations in the CRISPR/Cas9-targeted genes. The ease of

473 generating germline mutations using this approach will make it possible to test the function of

474 other predicted insect defense genes by knocking out their expression in the N. benthamiana

475 asat2-1 mutant background using a TRV-expressed gRNA.

476 Our knockout of acylsugar biosynthesis is an important first step toward improving the

477 already excellent $N$. benthamiana model system (Goodin et al., 2008; Bally et al., 2018), making

478 it more suitable for studying plant interactions with $M$. persicae, B. tabaci, and other

479 agriculturally relevant insect pests. Such experiments can include transient expression assays to

480 test the function of insect elicitors and insect-defensive genes from other plant species in $N$.

481 benthamiana, as well as VIGS to down-regulate insect gene expression in a targeted manner.

482 Furthermore, the almost complete absence of acylsugars in the asat 2 mutant lines, in

483 combination with the facile Agrobacterium- and virus-mediated transient gene expression

484 systems available for $N$. benthamiana, will make these mutants a suitable platform for the

485 functional analysis of ASATs from other Solanaceae.

487 Materials and Methods

488 Insect and plant cultures

489 A red strain of $M$. persicae (Ramsey et al., 2007; Ramsey et al., 2014), originally collected from

490 N. tabacum by Stewart Gray (Robert W. Holley Center for Agriculture \& Health, Ithaca, NY),

491 was maintained on $N$. tabacum plants in a growth room at $23^{\circ} \mathrm{C}$ with a $16: 8 \mathrm{~h}$ light:dark

492 photoperiod. Insect bioassays were conducted in the same growth room. Colonies of $B$. tabaci 
MEAM1 were provided by Danielle Preston and Angela Douglas (Cornell University) and Jane Polston (University of Florida). Macrosiphum euphorbiae was obtained from Isgouhi Kaloshian (UC Riverside) and was maintained on tomato cv. Moneymaker. Eggs of H. zea, H. virescens, and T. $n i$ were purchased from Benzon Research (www.benzonresearch.com). Nicotiana benthamiana wild type and mutant plants for aphid experiments, caterpillar experiments, and whitefly VIGS assays were maintained at $23^{\circ} \mathrm{C}$ and a $16: 8 \mathrm{~h}$ light:dark photoperiod in a Conviron (Winnipeg, Canada) growth chamber and, for seed production, in a greenhouse at $27 / 24^{\circ} \mathrm{C}$ (day/night) with ambient light conditions. Nicotiana benthamiana wild type and mutant plants for whitefly choice and no-choice assays were maintained at $26^{\circ} \mathrm{C}$ and a $16: 8 \mathrm{~h}$ light:dark photoperiod in a growth room and, for seed production, in a growth chamber (Percival Scientific, Perry, IA) at $27 / 24^{\circ} \mathrm{C}$ (day/night) with a $16: 8 \mathrm{~h}$ light:dark photoperiod.

\section{Identification of ASAT1 and ASAT2 orthologs in N. benthamiana}

To identify ASAT1 and ASAT2 orthologs in N. benthamiana, protein sequences of Salpiglossis sinuate and Solanum lycopersicum ASAT1 and ASAT2 (Moghe et al., 2017) were compared to predicted proteins encoded by the $N$. benthamiana genome. Sequences with $>67 \%$ identity were selected as potential ASAT1 and ASAT2 candidates and nucleotide sequences were obtained from the Solanaceae Genomics Network (www.solgenomics.net). The candidate ASAT sequences also were confirmed by comparing them to the most recent published $N$. benthamiana genome assembly (Schiavinato et al., 2019). To confirm the nucleotide sequences of $N$. benthamiana ASAT1 and ASAT2, genes were amplified with ASAT1F/ASAT1R and ASAT2F/ASAT2R primers (Table S4) using genomic DNA as the template. Amplified fragments were cloned in pDONOR ${ }^{\mathrm{TM}} 207$ (ThermoFisher Scientific, US) and were sequenced in their entirety using Sanger sequencing. This confirmatory sequencing showed no differences

520 A protein phylogenetic tree of previously annotated Solanaceae ASATs (Figures 2, S1, S2;

521 Tables S1, S2) was constructed using maximum likelihood method. Briefly, the ASATs protein 522 sequences were aligned in program ClustalW (Thompson et al., 1994). Then the alignment was 523 improved by removing the spurious sequences and poorly aligned regions (gap threshold at 0.25 ) 
using the program TrimAL v 1.4.rev22 (Capella-Gutierrez et al., 2009). Finally, an unrooted maximum likelihood tree was generated using the improved alignment with a bootstrap of 1000 in RAxML v8.2.12 (Stamatakis, 2014). The tree was visualized and presented using FigTree v1.4.4 (http://tree.bio.ed.ac.uk).

sgRNA design and plasmid cloning

Single-guide RNAs (sgRNA) targeting ASAT1 and ASAT2 were designed based on the coding regions using online software, CRISPR-P v2.0 (Liu et al., 2017) and CRISPRdirect (https://crispr.dbcls.jp/), based on two parameters, cleavage efficiency and potential off-targets. Additionally, only sgRNAs with $>40 \%$ GC content were selected. Three Cas9/gRNA constructs each were constructed for $A S A T 1$ and $A S A T 2$ following a previously developed CRISPR/Cas9 system (Jacobs et al., 2015). Four segments of DNA were prepared with 20 bp overlaps on their ends: ssDNA gRNA oligo, linearized p201N:Cas9 plasmid (Addgene 59175-59178), the Medicago truncatula (Mt) U6 promoter (377 bp), and a scaffold DNA (106 bp). For ssDNA gRNA, oligonucleotides targeting either the sense or antisense sequence of target genes were designed as: sense oligo TCAAGCGAACCAGTAGGCTT--GN19--

N19C-AAGCCTACTGGTTCGCTTGA. The gRNA oligonucleotide sequences are shown in

Figure 3 and Table S4.

Oligonucleotide sequences were synthesized by Integrated DNA Technologies

(www.idtdna.com). One $\mu \mathrm{l}$ of each $100 \mu \mathrm{M}$ oligo was added to $500 \mu \mathrm{l}$ 1x NEB buffer 2 (New Spe1 (www.neb.com) in $1 \mathrm{x}$ buffer 4 at $37^{\circ} \mathrm{C}$ for $2 \mathrm{~h}$, followed by column purification and a second digestion with $S w a l$ in 1 x buffer 3.1 at $25^{\circ} \mathrm{C}$ for $2 \mathrm{~h}$. Complete plasmid digestion was confirmed on a $0.8 \%$ agarose gel. The MtU6 promoter and Scaffold DNAs were PCR-amplified from the pUC gRNA Shuttle plasmid (Jacobs et al., 2015) using the primers Swal_MtU6F/MtU6R and ScaffoldF/Spe_ScaffoldR, respectively (Table S4). The PCR reactions were performed with a high-fidelity polymerase (2x Kapa master mix; www.sigmaaldrich.com) using the program: $95^{\circ} \mathrm{C}$ for $3 \mathrm{~min}$ followed by 31 cycles of $98^{\circ} \mathrm{C}$ for $20 \mathrm{sec}, 60^{\circ} \mathrm{C}$ for $30 \mathrm{sec}$, $72^{\circ} \mathrm{C}$ for $30 \mathrm{sec}$, and a final extension of $72^{\circ} \mathrm{C}$ for $5 \mathrm{~min}$. Finally, cloning was done using the NEBuilder® HiFi DNA Assembly Cloning Kit. For each reaction, the four pieces of DNA were 
mixed in a $20 \mu \mathrm{l}$ reaction with the NEBbuilder assembly mix with a final concentration of 0.011 pmol ( 100 ng) of p201N:Cas9 plasmid, 0.2 pmol of MtU6 amplicon ( $50 \mathrm{ng})$, scaffold amplicon $(\sim 12 \mathrm{ng})$ and ssDNA gRNA oligo (60-mer, $1 \mu \mathrm{l})$. The reactions were placed in thermal cycler at $50^{\circ} \mathrm{C}$ for $1 \mathrm{~h}$.

Two $\mu$ of the cloning reaction were transformed into $50 \mu$ of the One Shot ${ }^{\mathrm{TM}}$ Top10 chemically competent cells (Invitrogen, www.thermofisher.com) and plated on LB (Bertani, 1951) agar medium with $50 \mu \mathrm{l} / \mathrm{ml}$ kanamycin for selection of transformants. Colonies with the correct inserts were screened using the Ubi3p218R and IScelR primers (Table S4). Plasmids carrying the designed gRNA constructs were then transformed into Agrobacterium tumefaciens strain GV3101 for generating transgenic plants. All constructs were confirmed by Sanger sequencing.

To avoid off-target effects, gRNAs were further checked by comparison against the reference $N$. benthamiana genome v1.0.1 (www.solgenomics.net). Only two sites in the $N$. benthamiana genome were found to have non-target matches $>17$ nt (both with 1 internal mismatch), and with the NGG PAM sequence on the correct strand. These two sites were checked by PCR amplification and Sanger sequencing and showed no unexpected editing in our asat1-1, asat1-2, asat2-1, or asat2-2 mutant plants. Primers used for off-target Sanger sequencing are listed in Table S4.

575 For both ASAT1 and ASAT2, we Agrobacterium-infiltrated N. benthamiana plants at the four-leaf stage. For ASAT1, we used Agrobacterium carrying the Cas9/gRNA constructs at OD of 1 and 3,

577 whereas for ASAT2, we used an OD 1.5. Each leaf was saturated with Agrobacterium solution.

578 After infiltration, the plants were cultured in a growth chamber for 2 days and then the infiltrated

579 whole leaves were collected for genomic DNA extraction and tested by PCR amplification for

580 detection of insertion/deletion polymorphisms in the target region (Figure S9). During the

581 method optimization, a positive control construct targeting the $N$. benthamiana Drm3 gene

582 (gRNA: GCCACTATCTGGCCGGGGAC, provided by the Greg Martin lab, Boyce Thompson

583 Institute) was infiltrated in parallel. 
Stable ASAT mutant $N$. benthamiana plants were created in the Boyce Thompson Institute plant transformation facility using CRISPR/Cas9 with gRNAs that had been confirmed to be functional as described above and a previously described protocol (Van Eck et al., 2019), with minor modifications. To prepare plants for transformation, we disinfected $N$. benthamiana seeds with $1.5 \mathrm{ml} 1.25 \%$ sodium hypochlorite (1:5 dilution of $5.25 \%$ sodium hypochlorite Clorox bleach), with $100 \mu \mathrm{l}$ Tween-20 added, for $20 \mathrm{~min}$ on a shaker platform. After sodium

592 hypochlorite treatment, seeds were rinsed three times with sterile water. Subsequently, we placed 593 seeds on Tobacco Seed Germination Medium (composition per liter: $1.08 \mathrm{~g}$ Murashige and 594 Skoog salts (Murashige and Skoog, 1962), $30 \mathrm{~g}$ sucrose, and $8 \mathrm{~g}$ agar, $\mathrm{pH}=5.7 \pm 0.1$ ) and 595 incubated the seeds under yellow filtered light with a 16 -h photoperiod at $27^{\circ} \mathrm{C}$. After $\sim 2$ weeks, 596 the seedlings were transferred to the Rooting Medium (composition per liter: $2.15 \mathrm{~g}$ Murashige and Skoog salts, $30 \mathrm{~g}$ sucrose, $1 \mathrm{ml} \mathrm{B5}$ vitamin/amino acid stock, and $8 \mathrm{~g}$ agar, $\mathrm{pH}=5.7 \pm 0.1$ ). After $\sim 6$ weeks, the plants were ready for Agrobacterium infection. First, Agrobacterium carrying the gRNA plasmids was amplified in YEP medium (composition per liter: $10 \mathrm{~g}$ yeast extract, $10 \mathrm{~g}$ peptone, and $5 \mathrm{~g} \mathrm{NaCl}$ with antibiotics, $50 \mu \mathrm{g} / \mu \mathrm{l}$ gentamycin, $50 \mu \mathrm{g} / \mu \mathrm{l}$ kanamycin, and $50 \mu \mathrm{g} / \mu \mathrm{l}$ rifampicin), and Agrobacterium culture then was pelleted and combined to a certain

602 OD (OD 1.0 for ASAT1 gRNAs, and OD 1.0 or 1.5 for ASAT2 gRNAs) with filtered cell 603 suspension buffer (10 mM MES, $10 \mathrm{mM} \mathrm{MgCl} 2$ and $200 \mu \mathrm{M}$ acetosyringone). Then, fully

604 expanded but immature $N$. benthamiana leaves were dissected into $5 \mathrm{~mm}$ segments by removing 605 the leaf margins. The leaf segments were incubated in the Agrobacterium culture for 30 min with 606 shaking. The incubated leaf segments were transferred to co-cultivation medium plates

607 (composition per liter: $4.3 \mathrm{~g}$ Murashige and Skoog salts, $30 \mathrm{~g}$ sucrose, $1 \mathrm{ml}$ B5 vitamin/amino 608 acid stock, and $5 \mathrm{~g}$ agar, $\mathrm{pH}=5.7 \pm 0.1$ ) and kept in the dark at room temperature. Leaf segments 609 treated with YEP medium were used as a negative control. After three days, the leaf segments 610 were transferred from co-cultivation medium to shoot bud initiation medium with selections 611 (composition per liter: $4.3 \mathrm{~g}$ Murashige and Skoog salts, $30 \mathrm{~g}$ sucrose, $1 \mathrm{ml}$ B5 vitamin/amino 612 acid stock, $0.1 \mathrm{mg}$ NAA, $1.0 \mathrm{mg} \mathrm{BA}$, and $6 \mathrm{~g}$ agar, $\mathrm{pH}=5.7 \pm 0.1$; with $200 \mu \mathrm{g} / \mathrm{ml}$ kanamycin and $613250 \mu \mathrm{g} / \mathrm{ml}$ Timentin). Medium with Timentin but without kanamycin was used as a positive 614 control. Leaf segments on selection medium were incubated under a 16:8 h light-dark 615 photoperiod at $24-25^{\circ} \mathrm{C}$ under yellow filtered light. The leaf segments were sub-cultured every 2616 weeks onto the same medium until shoot buds were visible. Finally, to generate roots, shoot buds 
617 were transferred to rooting medium (composition per liter: $2.15 \mathrm{~g}$ Murashige and Skoog salts, 30

$618 \mathrm{~g}$ sucrose, $1 \mathrm{ml} \mathrm{B5}$ vitamin/amino acid stock, $8 \mathrm{~g}$ agar with $200 \mu \mathrm{g} / \mathrm{ml}$ kanamycin and $250 \mu \mathrm{g} / \mathrm{ml}$

619 Timentin, $\mathrm{pH}=5.7 \pm 0.1)$.

620 Confirmation of homozygous mutant plants in the T2 generation

621 Rooted N. benthamiana plants from tissue culture were transferred to soil (T0 generation).

622 CRISPR/Cas9-induced mutations were identified by PCR amplification of genomic regions of

623 the gRNA target sites in ASAT1 and ASAT2 (Figure 3), followed by Sanger sequencing. Lines

624 with mutations were used to generate T1 plants, which were subjected to PCR amplification and

625 sequencing to confirm homozygous mutations. T2 seeds from confirmed homozygous mutant

626 asat1-1, asat1-2, asat2-1, and asat2-2 T1 plants were used for all experiments. Homozygous

627 mutations were confirmed in randomly selected T2 plants by PCR amplification and Sanger

628 sequencing. The presence of Cas9 in transgenic plants in the T0, T1, and T2 generations was

629 confirmed by PCR amplification and agarose gel electrophoresis. Primers that were used to

630 identify mutations and confirm the presence of Cas9 are listed in Table S4.

632 Acylsugar measurements by LC/MS

633 Liquid chromatography/mass spectrometry (LC/MS) was used to confirm the effect of the $A S A T$

634 mutations by measuring acylsugar content in leaf extracts from wildtype and ASAT mutant

635 plants. New leaflets were rinsed in acylsugar extraction solution (3:3:2

636 acetonitrile:isopropanol:water, $0.1 \%$ formic acid, and $1 \mu \mathrm{M}$ Telmisartan as internal standard) and

637 gently agitated for $2 \mathrm{~min}$. Then, the extraction solutions were transferred to LC/MS glass vials,

638 and the leaves were air dried for leaf weight measurements.

639 Chromatography of leaf surface washes was performed on a ThermoScientific Ultimate

6403000 HPLC with a glass vial autosampler and coupled with a Thermo Scientific Q Exactive ${ }^{\mathrm{TM}}$

641 Hybrid Quadrupole-Orbitrap ${ }^{\mathrm{TM}}$ Mass Spectrometer (Mass Spectrometry Facility at Boyce

642 Thompson Institute). Acylsugar extracts were separated on an Ascentis Express C18 HPLC

643 column $(10 \mathrm{~cm} \times 2.1 \mathrm{~mm} \times 2.7 \mu \mathrm{m})($ Sigma $\square$ Aldrich, St. Louis, MO) with a flow rate of 0.3

$644 \mathrm{ml} / \mathrm{min}$, using a gradient flow of $0.1 \%$ formic acid (Solvent A) and 100\% acetonitrile (Solvent

645 B). We used a 7-min LC method for metabolite profiling, which involved a linear gradient from

646 95:5 A:B to 0:98 A:B. Full $\square$ scan mass spectra were collected (mass range: m/z 50-1000) in both

647 positive and negative electron spray ionization (ESI) modes. Mass spectral parameters were set 
648 as follows: capillary spray voltage $2.00 \mathrm{kV}$ for negative ion-mode and $3.00 \mathrm{kV}$ for positive ion-

649 mode, source temperature: $100^{\circ} \mathrm{C}$, desolvation temperature $350^{\circ} \mathrm{C}$, desolvation nitrogen gas flow

650 rate: 600 liters/h, cone voltage $35 \mathrm{~V}$. Acylsugars were identified and annotated using Thermo

651 Xcalibur Qual Browser (Thermo Fisher) and MS-DIAL v4.20 based on the MS/MS peak features

652 and neutral losses. The acylsugar abundances were estimated using peak areas at the respective

$653 \mathrm{~m} / \mathrm{z}$ channel under negative ESI mode. Acylsugar quantification was first normalized to the

654 internal control Telmisartan to account for technical variation between samples, and then

655 normalized to the leaf dry weight to allow comparisons between samples.

Insect choice and no-choice bioassays

658 To measure M. persicae and M. euphorbiae growth, we caged aphids on individual leaves of 659 mutant and wildtype 4 to 5-week-old $N$. benthamiana (Figure S10A, B). Twenty adult $M$.

660 persicae from N. tabacum (naïve to N. benthamiana) were placed in each cage and allowed to 661 generate nymphs for $\sim 12$ hrs. Twenty-five nymphs were left in each cage and were monitored for $6625 \mathrm{~d}$ to assess nymph survival. At the end of the $M$. persicae survival monitoring period, five $M$.

663 persicae were left in each cage and reproduction was monitored for one week. Finally, the

664 remaining $M$. persicae were collected to measure aphid size by taking a picture and assessing the 665 area of each aphid using ImageJ (Schneider et al., 2012). Ten adult M. euphorbiae from a colony

666 on tomato cv. Moneymaker were placed in each individual cage on N. benthamiana leaves.

667 Surviving aphids and progeny were counted after 24 hours.

668 Myzus persicae and M. euphorbiae choice assays were performed with detached leaves

669 from 4 to 5-week-old N. benthamiana. Two similarly-sized leaves from individual ASAT mutant

670 and wildtype plants were cut and placed in 15-cm Petri dishes, with their petioles inserted in

671 moistened cotton swabs (pairwise comparison is shown in Figure S10C). Ten naïve adult aphids,

672 which had not previously encountered $N$. benthamiana, were released at the midpoint between

673 pairs of leaves (wildtype, asat1, or asat2), and the Petri dishes were placed under 16:8 h

674 light:dark photoperiod. The aphids on each leaf were counted at $24 \mathrm{~h}$ after their release in the

675 Petri dishes.

676 To measure whitefly survival and fecundity on wildtype and asat2-1 N. benthamiana

677 plants, cages were set up with $N$. benthamiana plants at the seven-leaf stage (approximately 3

678 weeks old). Each cage contained three plants, either wildtype or asat2-1. Ninety adult whiteflies 
679 reared on Brassica oleracea (variety Earliana; www.burpee.com, catalog number 62729A) were

680 introduced into each cage $(60 \times 60 \times 60 \mathrm{~cm})$ with $N$. benthamiana $(30$ whiteflies/plant $)$ and were

681 allowed to feed for three days at $26^{\circ} \mathrm{C}$ with a $16: 8 \mathrm{~h}$ light:dark photoperiod. The numbers of

682 whiteflies surviving on each host plant were counted, after which the remaining insects were

683 killed with insecticidal soap. The following day, the number of whitefly eggs on each plant was

684 counted. This experiment was conducted twice with similar results.

685 For whitefly choice assays, wildtype and asat2-1 plants at the seven-leaf stage were

686 placed together in the same cage. Approximately 150 whiteflies from cabbage plants were

687 moved into each cage. After $24 \mathrm{~h}$ at $26^{\circ} \mathrm{C}$ with a $16: 8 \mathrm{~h}$ light:dark photoperiod, live and dead

688 whiteflies were counted on the plants and elsewhere in the cage. This experiment was repeated

689 three times.

690 Eggs of H. zea, H. virescens and T. ni were hatched on artificial diet (Southland Products,

691 Lake Village, Arkansas). Neonate larvae were confined onto individual N. benthamiana leaves,

692 one larva per plant, using 10 x $15 \mathrm{~cm}$ organza mesh bags (www.amazon.com, item

693 B073J4RS9C). After ten days, the surviving larvae were counted and weighed. In a separate

694 experiment, H. zea, H. virescens, and T. ni were reared on artificial diet (beet armyworm diet,

695 www.southlandproducts.net) for five days. Individual five-day-old caterpillars were weighed and

696 then confined on 4 to 4.5-week-old $N$ benthamiana plants using $30 \mathrm{~cm}$ x $60 \mathrm{~cm}$ micro-perforated

697 bread bags (www.amazon.com). After seven days, the surviving larvae were weighed again.

698 Relative growth rate was calculated as: $\ln ((($ day-12 mass $) /($ mean day-5 mass $)) / 7)$.

Leaf water loss and temperature assays

701 To measure the leaf water loss, two leaves from each of eight plants were detached. The fresh

702 weight of each leaf was determined on a Sartorius Ultra Micro Balance. All leaves were placed

703 at $23^{\circ} \mathrm{C}$ and a 16:8 h light:dark photoperiod. Each leaf was weighted again after $24 \mathrm{~h}$ and the

704 percentage of water loss was calculated as [(fresh weight - final weight)/fresh weight]*100\%.

705 Thermal images were acquired in the growth chamber environment using a thermal

706 camera (A655sc, FLIR Systems Inc., Boston, MA, USA) with a spectral range of 7.5-14.0 mm

707 and a resolution of 640 x 480 pixels. The camera was placed approximately $1 \mathrm{~m}$ away from each

708 plant and a white background was used when the plant images were acquired. One region of

709 interest, corresponding to the perimeter of each leaf, was specified per leaf for each of 20 leaves 
710 per genotype. Using the FLIR ResearchIR Max software (version 4.40.9.30) thermal images files

711 were exported as CSV files. Images were segmented from the background using Gaussian

712 mixture models in MATLAB to determine the temperature of each leaf. After segmentation, the

713 temperature was averaged across the segmented leaf.

714 Hyperspectral images were acquired in a dark room using a hyperspectral imager

715 (SOC710, Series 70-V, Surface Optics Corporation, San Diego, CA, USA) that covered a

716 spectral range from 400 to $1000 \mathrm{~nm}$ for 128 wavebands. Image acquisition and recording were

717 performed using a Dell DELL XPS 159570 laptop computer that controls the camera. The

718 camera was fixed using a stand with the lens facing the plants and capturing top view images

719 approximately $1 \mathrm{~m}$ away. A Spectralon tile (Labsphere, Inc, North Sutton NH, USA) was placed

720 next to the plant trays, covering one corner of the image to facilitate subsequent image

721 processing and calibration. The nominal reflectance value for the Spectralon tile was $99 \%$ and it

722 had a $30.5 \mathrm{~cm}$ x $30.5 \mathrm{~cm}$ reflective area. Lighting consisted of two halogen lamps placed at $\sim 45^{\circ}$

723 angles on either side of the camera to create an even light distribution. All image analysis was

724 performed in HSIviewer, a MATLAB package (Stone et al., 2020). White reflectance calibration

725 was performed using the Spectralon tile. One region of interest ROI was specified for each of 20

726 leaves per $N$. benthamiana genotype. This ROI corresponded to the perimeter of each leaf. From

727 each hyperspectral cube image, the vegetation pixels (green portion of the plant) were extracted

728 using the Normalized Difference Vegetation Index (NDVI). Mean reflectance (R) was calculated

729 per band per 10 leaves in order to obtained the WBI results

730 To calculate NDVI and WBI we used the following formulas, where $R$ corresponds to the

731 reflectance at a specific wavelength $(\mathrm{nm})$ : WBI = (R970/R900) (Penuelas et al., 1993) and NVDI

$732=(\mathrm{R} 750-\mathrm{R} 705) /(\mathrm{R} 750+\mathrm{R} 705)($ Gitelson and Merzlyak, 1994).

734 Virus transmission assays

735 For virus transmission assays, young adult $M$. persicae were starved for $3 \mathrm{~h}$ and then allowed to

736 feed for 30 min on 6-week-old wildtype or asat2-1 N. benthamiana plants infected with TuMV-

737 GFP (Lellis et al., 2002; Casteel et al., 2015). Aphids were transferred in groups of ten to

738 individual 4-week-old wildtype or asat2-1 recipient plants. The aphids were allowed to feed on

739 recipient plants for $24 \mathrm{~h}$ and were then removed. Donor and recipient plants were kept in the

740 Conviron growth chamber at $23^{\circ} \mathrm{C}$ and a $16: 8 \mathrm{~h}$ light:dark photoperiod. The development of GFP 
741 fluorescence was monitored every $24 \mathrm{~h}$ using a $395 \mathrm{~nm}$ UV flashlight (www.walmart.com) in the

742 dark, until no new GFP signals developed from one day to the next. In each experiment, the virus

743 donor plant group consisted of eight TuMV-GFP infected plants ( 2 weeks after TuMV-GFP

744 infection by rub-inoculation) of each genotype, with aphids randomly spread on the plants. Each

745 recipient group consisted of 20 plants of each genotype, with the aphids confined on individual

746 plants. The experiment was repeated three times with similar results, and the results were

747 combined for statistical analysis of differences between groups using one-way ANOVA with a

748 block effect followed by a Tukey HSD post hoc test.

Plant-mediated virus induced gene silencing in whitefly with WT and asat2-1

751 For each target gene, a 200-550 bp RNA sequence was designed using the E-RNA $i$ webserver

752 (https://www.dkfz.de/signaling/e-rnai3/). The designed fragments were checked against the $B$.

753 tabaci MEAM1 (Chen et al., 2016) and N. benthamiana (Bombarely et al., 2012) reference

754 genomes using local blast (Mount, 2007) to avoid potential off-target sequences with full

755 matches $\geq 19$ nt. The identified gene fragments were then PCR-amplified from whitefly cDNA

756 and cloned into a binary tobacco rattle virus VIGS vector pTRV2 (Liu et al., 2002) using the

757 Invitrogen Gateway recombination cloning technology (Invitrogen, USA). As a positive control,

758 we used a pTRV2-PDS construct with an RNA fragment targeting $N$. benthamiana phytoene

759 desaturase (Velásquez et al., 2009). As negative controls, we used pTRV2-EV (empty vector)

760 and pTRV2-GFP, with an RNA fragment targeting Aequorea victoria GFP, a sequence that is

761 not present in either $N$. benthamiana or $B$. tabaci. The PCR primers used to generate the VIGS

762 vectors are in Supplemental Table S4.

763 To infiltrate plants, plasmids (pTRV1 and pTRV2 containing constructs targeting the

764 genes of interest) were transformed into Agrobacterium tumefaciens GV3101. Equal amounts of

765 Agrobacterium with TRV1 and TRV2 were resuspended in infiltration medium (10 mM MES,

$76610 \mathrm{mM} \mathrm{MgCl}_{2}, 200 \mu \mathrm{M}$ acetosyringone) to an OD 0.3 and incubated at room temperature for $3 \mathrm{~h}$.

767 The Agrobacterium mixture was infiltrated using a 1-ml needleless syringe to saturate three

768 leaves of $\sim 4$-week-old $N$. benthamiana. Inoculated plants were kept in a Conviron growth

769 chamber for 2 3 weeks until control plants infiltrated with pTRV2-PDS showed photobleaching

770 symptoms. Successful growth of TRV targeting the genes of interest in whiteflies was confirmed

771 by PCR and the confirmed plants were then used for insect bioassays. 
For whitefly bioassays, three infiltrated plants were used for each gene of interest, and

773 three cages were attached to each plant. Ten young adult whiteflies (emerged within the past

774 week) were placed in each cage. After seven days, the survival rates of whiteflies were assessed

775 and compared across different treatments. The surviving whiteflies were collected for qPCR

776 analyses of target gene expression using quantitative PCR using the PowerUp ${ }^{\mathrm{TM}} \mathrm{SYBR}^{\mathrm{TM}}$ green

777 Master Mix (AppliedBiosystem, Thermo Fisher Scientific) on a QuantStudio ${ }^{\mathrm{TM}} 6$ Flex Real-time

778 PCR system (AppliedBiosystem, Thermo Fisher Scientific), and results were analyzed using the

$7792^{-\triangle \Delta C T}$ method (Schmittgen and Livak, 2008). The whitefly VIGS bioassays, comparing asat2-1

780 and wildtype $N$. benthamiana, were repeated three times with similar results. Two replicates of a

781 separate experiment was set up on asat2-1 plants to collect whiteflies after one day for gene

782 expression analyses.

784 Statistical analysis

785 All statistical comparisons were conducted using SPSS v25, R and MATLAB R2019a

786 (MathWorks, Inc., Natick, MA, USA). ANOVA followed by a Dunnett's post hoc test was used

787 to determine differences in leaf water loss, leaf temperature, and WBI across genotypes in each

788 data set. ANOVA followed by a Duncan post hoc test was used for aphid bioassay and LC/MS

789 results. A Chi-square test was used to test for differences in pairwise aphid choice assays,

790 whitefly, and lepidopteran assays.

\section{Acknowledgements}

793 We want to thank Patricia Keen and Joyce Van Eck for their help with N. benthamiana stable

794 transformation and access to the tissue culture facility, Danielle Preston, Angela Douglas, and

795 Jane Polston for providing whitefly colonies, Isgouhi Kaloshian for the potato aphid colony,

796 Ning Zhang and Greg Martin for sharing the p201N-Cas9 construct and the Drm3 sgRNA

797 control construct, and William Stone and Thomas Lawton for providing custom image

798 processing software. This research was supported by Cornell startup funds to G.D.M., Deutsche

799 Forschungsgemeinschaft award \#411255989 to L.H.K., and United States Department of

800 Agriculture Biotechnology Risk Assessment Grant 2017-33522-27006, US National Science

801 Foundation award IOS-1645256, and Defense Advanced Research Projects Agency (DARPA)

802 agreement HR0011-17-2-0053 to G.J, and US National Science Foundation award \#1723926 to 
803 C.L.C. G.S. is part of a team supporting DARPA's Insect Allies program under agreement

804 HR0011-17-2-0055. M.A.G. is part of a team supporting DARPA's Advanced Plant

805 Technologies program under agreement HR0011-18-C-0146. The views and conclusions

806 contained in this document are those of the authors and should not be interpreted as representing

807 the official policies of the U.S. Government.

\section{Conflict of interest}

810 The authors declare that there is no conflict of interest.

\section{Author Contributions}

813 G.J. and H.F. conceived the original research plans; H.F., S.S., L.A., H.X., L.K., J.D.T, S.H.C., 814 and A.N.F. performed the experiments; H.F., L.A., L.K., and G.D.M. analyzed the data; C.L.C., 815 M.A.G., G.D.M., G.S., and G.J. supervised the experiments; H.F. and G.J. wrote the article with 816 contributions from all of the authors; G.J. agrees to serve as the contact author responsible for 817 communication and distribution of samples.

\section{References}

820 Alba JM, Montserrat M, Fernandez-Munoz R (2009) Resistance to the two-spotted spider mite (Tetranychus urticae) by acylsucroses of wild tomato (Solanum pimpinellifolium) trichomes studied in a recombinant inbred line population. Exp Appl Acarol 47: 35-47

Arntzen C (2015) Plant-made pharmaceuticals: from 'Edible Vaccines' to Ebola therapeutics. Plant Biotechnology Journal 13: 1013-1016

825 Arrendale RF, Severson RF, Sisson VA, Costello CE, Leary JA, Himmelsbach DS, Vanhalbeek H (1990) Characterization of the sucrose ester fraction from Nicotiana glutinosa. Journal of Agricultural and Food Chemistry 38: 75-85

828 Bally J, Jung H, Mortimer C, Naim F, Philips JG, Hellens R, Bombarely A, Goodin MM, Waterhouse PM (2018) The rise and rise of Nicotiana benthamiana: a plant for all

831 Bertani G (1951) Studies on lysogenesis. I. The mode of phage liberation by lysogenic Escherichia coli. J Bacteriol 62: 293-300 
Bombarely A, Rosli HG, Vrebalov J, Moffett P, Mueller LA, Martin GB (2012) A draft genome sequence of Nicotiana benthamiana to enhance molecular plant-microbe biology research. Mol Plant-Micr Int 25: 1523-1530

Bos JIB, Prince D, Pitino M, Maffei ME, Win J, Hogenhout SA (2010) A functional genomics approach identifies candidate effectors from the aphid species Myzus persicae (green peach aphid). Plos Genetics 6: e1001216.

Cardoso MZ (2008) Herbivore handling of a plant's trichome: The case of Heliconius charithonia (L.) (Lepidoptera : Nymphalidae) and Passiflora Lobata (Killip) Hutch. (Passifloraceae). Neotropical Entomology 37: 247-252

Casteel CL, De Alwis M, Bak A, Dong H, Whitham SA, Jander G (2015) Disruption of

Casteel CL, Yang CL, Nanduri AC, De Jong HN, Whitham SA, Jander G (2014) The NIaPro protein of Turnip mosaic virus improves growth and reproduction of the aphid vector, Myzus persicae (green peach aphid). Plant Journal 77: 653-663

Chen W, Hasegawa DK, Kaur N, Kliot A, Pinheiro PV, Luan J, Stensmyr MC, Zheng Y, Liu W, Sun H, Xu Y, Luo Y, Kruse A, Yang X, Kontsedalov S, Lebedev G, Fisher TW, Nelson DR, Hunter WB, Brown JK, Jander G, Cilia M, Douglas AE, Ghanim M, Simmons AM, Wintermantel WM, Ling KS, Fei Z (2016) The draft genome of whitefly Bemisia tabaci MEAM1, a global crop pest, provides novel insights into virus transmission, host adaptation, and insecticide resistance. BMC Biol 14: 110

Chen X, Li L, Hu Q, Zhang B, Wu W, Jin F, Jiang J (2015) Expression of dsRNA in recombinant Isaria fumosorosea strain targets the TLR7 gene in Bemisia tabaci. BMC Biotechnol 15: 64

Chortyk OT, Pomonis JG, Johnson AW (1996) Syntheses and characterizations of insecticidal sucrose esters. J Ag Food Chem 44: 1551-1557

Clay N, Adio A, Denoux C, Jander G, Ausubel F (2009) Glucosinolate metabolites required for an Arabidopsis innate immune response. Science 323: 95-101 
Cui N, Lu H, Wang TZ, Zhang WH, Kang L, Cui F (2019) Armet, an aphid effector protein, induces pathogen resistance in plants by promoting the accumulation of salicylic acid. Philosophical Transactions of the Royal Society B-Biological Sciences 374

D'Auria JC (2006) Acyltransferases in plants: a good time to be BAHD. Curr Opin Plant Biol 9: 331-340

Del Toro FJ, Mencia E, Aguilar E, Tenllado F, Canto T (2018) HCPro-mediated transmission

Egan AN, Moore S, Stellari GM, Kang BC, Jahn MM (2019) Tandem gene duplication and recombination at the AT3 locus in the Solanaceae, a gene essential for capsaicinoid biosynthesis in Capsicum. Plos One 14: e0210510 Multiplexed heritable gene editing using RNA viruses and mobile single guide RNAs. Nat Plants 6: 620-624

Elzinga DA, De Vos M, Jander G (2014) Suppression of plant defenses by a Myzus persicae (green peach aphid) salivary effector protein. Mol Plant Microbe Interact 27: 747-756

Fan P, Miller AM, Schilmiller AL, Liu X, Ofner I, Jones AD, Zamir D, Last RL (2016) In vitro reconstruction and analysis of evolutionary variation of the tomato acylsucrose metabolic network. Proc Natl Acad Sci U S A 113: E239-248

889 Gaquerel E, Kotkar H, Onkokesung N, Galis I, Baldwin IT (2013) Silencing an Nacyltransferase-like involved in lignin biosynthesis in Nicotiana attenuata dramatically alters herbivory-induced phenolamide metabolism. Plos One 8: e62336 
892

893

894

895

896

897

898

899

900

901

902

903

904

905

906

907

908

909

910

911

912

913

914

915

916

917

918

919

920

921

Gitelson A, Merzlyak MN (1994) Spectral reflectance changes associated with autumn senescence of Aesculus hippocastanum $\mathrm{L}$ and Acer platanoides $\mathrm{L}$ leaves - spectral features and relation to chlorophyll estimation. J Plant Physiol 143: 286-292

Glas JJ, Schimmel BCJ, Alba JM, Escobar-Bravo R, Schuurink RC, Kant MR (2012) Plant glandular trichomes as targets for breeding or engineering of resistance to herbivores. International Journal of Molecular Sciences 13: 17077-17103

Gong P, Zhang J, Li H, Yang C, Zhang C, Zhang X, Khurram Z, Zhang Y, Wang T, Fei Z, Ye Z (2010) Transcriptional profiles of drought-responsive genes in modulating transcription signal transduction, and biochemical pathways in tomato. J Exp Bot 61: 3563-3575

Goodin MM, Zaitlin D, Naidu RA, Lommel SA (2008) Nicotiana benthamiana: its history and future as a model for plant-pathogen interactions. Mol Plant Microbe Interact 21: 10151026

Hagimori M, Matsui M, Matsuzaki T, Shinozaki Y, Shinoda T, Harada H (1993) Production of somatic hybrids between Nicotiana benthamiana and Nicotiana tabacum and their resistance to aphids. Plant Science 91: 213-222

Hayward A, Padmanabhan M, Dinesh-Kumar SP (2011) Virus-induced gene silencing in Nicotiana benthamiana and other plant species. Plant Reverse Genetics: Methods and Protocols 678: 55-63

Jacobs TB, LaFayette PR, Schmitz RJ, Parrott WA (2015) Targeted genome modifications in soybean with CRISPR/Cas9. Bmc Biotechnology 15: 16

Kandra L, Wagner GJ (1988) Studies of the site and mode of biosynthesis of tobacco trichome exudate components. Archives of Biochemistry and Biophysics 265: 425-432

Karabourniotis G, Kotsabassidis D, Manetas Y (1995) Trichome density and its protective potential against ultraviolet-B radiation damage during leaf development. Canadian Journal of Botany-Revue Canadienne De Botanique 73: 376-383

Kim J, Kang K, Gonzales-Vigil E, Shi F, Jones AD, Barry CS, Last RL (2012) Striking natural diversity in glandular trichome acylsugar composition is shaped by variation at the acyltransferase2 locus in the wild tomato Solanum habrochaites. Plant Physiol 160: $1854-1870$ 
922 Krenz B, Bronikowski A, Lu XY, Ziebell H, Thompson JR, Perry KL (2015) Visual monitoring of Cucumber mosaic virus infection in Nicotiana benthamiana following transmission by the aphid vector Myzus persicae. Journal of General Virology 96: 29042912

Kroumova AB, Wagner GJ (2003) Different elongation pathways in the biosynthesis of acyl groups of trichome exudate sugar esters from various solanaceous plants. Planta 216: 1013-1021

Kroumova ABM, Zaitlin D, Wagner GJ (2016) Natural variability in acyl moieties of sugar esters produced by certain tobacco and other Solanaceae species. Phytochemistry 130: 218-227

Laue G, Preston CA, Baldwin IT (2000) Fast track to the trichome: induction of N-acyl nornicotines precedes nicotine induction in Nicotiana repanda. Planta 210: 510-514

Lellis AD, Kasschau KD, Whitham SA, Carrington JC (2002) Loss-of-susceptibility mutants of Arabidopsis thaliana reveal an essential role for eIF(iso)4E during potyvirus infection. Curr Biol 12: 1046-1051

Leong BJ, Lybrand DB, Lou YR, Fan PX, Schilmiller AL, Last RL (2019) Evolution of metabolic novelty: A trichome-expressed invertase creates specialized metabolic diversity in wild tomato. Science Advances 5: eaaw3754

Liu H, Ding YD, Zhou YQ, Jin WQ, Xie KB, Chen LL (2017) CRISPR-P 2.0: an improved CRISPR-Cas9 tool for genome editing in plants. Molecular Plant 10: 530-532

Liu Y, Schiff M, Dinesh-Kumar SP (2002) Virus-induced gene silencing in tomato. Plant J 31: 777-786

Malik HJ, Raza A, Amin I, Scheffler JA, Scheffler BE, Brown JK, Mansoor S (2016) RNAimediated mortality of the whitefly through transgenic expression of double-stranded RNA homologous to acetylcholinesterase and ecdysone receptor in tobacco plants. Sci Rep 6: 38469

Mathers TC, Chen Y, Kaithakottil G, Legeai F, Mugford ST, Baa-Puyoulet P, Bretaudeau A, Clavijo B, Colella S, Collin O, Dalmay T, Derrien T, Feng H, Gabaldon T, Jordan A, Julca I, Kettles GJ, Kowitwanich K, Lavenier D, Lenzi P, LopezGomollon S, Loska D, Mapleson D, Maumus F, Moxon S, Price DR, Sugio A, van Munster M, Uzest M, Waite D, Jander G, Tagu D, Wilson ACC, van Oosterhout C, 
Swarbreck D, Hogenhout SA (2017) Rapid transcriptional plasticity of duplicated gene clusters enables a clonally reproducing aphid to colonise diverse plant species. Genome Biology 18: 27

Matsuzaki T, Shinozaki Y, Hagimori M, Tobita T, Shigematsu H, Koiwai A (1992) Novel glycerolipids and glycolipids from the surface-lipids of Nicotiana benthamiana. Bioscience Biotechnology and Biochemistry 56: 1565-1569 Isolation of glycolipids from the surface-lipids of Nicotiana bigelovii and their distribution in Nicotiana species. Agricultural and Biological Chemistry 53: 3079-3082

McKenzie CL, Puterka GJ (2004) Effect of sucrose octanoate on survival of nymphal and adult Diaphorina citri (Homoptera : Psyllidae). J Econ Entomol 97: 970-975

McKenzie CL, Weathersbee AA, 3rd, Puterka GJ (2005) Toxicity of sucrose octanoate to egg, nymphal, and adult Bemisia tabaci (Hemiptera: Aleyrodidae) using a novel plantbased bioassay. J Econ Entomol 98: 1242-1247

Meihls LN, Handrick V, Glauser G, Barbier H, Kaur H, Haribal MM, Lipka AE,

Moghe GD, Leong BJ, Hurney SM, Jones AD, Last RL (2017) Evolutionary routes to biochemical innovation revealed by integrative analysis of a plant-defense related

974 Mount DW (2007) Using the basic local alignment search tool (BLAST) Cold Spring Harbor Protocols doi:10.1101/pdb.top17

Mulot M, Boissinot S, Monsion B, Rastegar M, Clavijo G, Halter D, Bochet N, Erdinger M, Brault V (2016) Comparative analysis of RNAi-based methods to down-regulate expression of two genes expressed at different levels in Myzus persicae. Viruses 8: 316

Murashige T, Skoog FA (1962) A revised medium for rapid growth and bioassays with tobacco tissue cultures. Physiol Plant 15: 473-497

\section{Ning J, Moghe GD, Leong B, Kim J, Ofner I, Wang Z, Adams C, Jones AD, Zamir D, Last} RL (2015) A feedback-insensitive isopropylmalate synthase affects acylsugar composition in cultivated and wild tomato. Plant Physiol 169: 1821-1835 
O' Connell MA, Medina AL, Sanchez Pena Pand Trevino MB (2007) Molecular genetics of drought resistance response in tomato and related species. In Razdan, M. K., \& Mattoo, A. K. (eds). Genetic Improvement of Solanaceous Crops 2: Enfield USA 261-283

Penuelas J, Filella I, Biel C, Serrano L, Save R (1993) The reflectance at the 950-970 nm region as an indicator of plant water status. International Journal of Remote Sensing 14:

Powell JD (2015) From pandemic preparedness to biofuel production: tobacco finds its biotechnology niche in North America. Agriculture-Basel 5: 901-917

Ramsey JS, Elzinga DA, Sarkar P, Xin YR, Ghanim M, Jander G (2014) Adaptation to nicotine feeding in Myzus persicae. J Chem Ecol 40: 869-877

Ramsey JS, Wilson AC, De Vos M, Sun Q, Tamborindeguy C, Winfield A, Malloch G, Smith DM, Fenton B, Gray SM, Jander G (2007) Genomic resources for Myzus persicae: EST sequencing, SNP identification, and microarray design. BMC Genomics 8: 423

Rodriguez AE, Tingey WM, Mutschler MA (1993) Acylsugars of Lycopersicon pennellii deter settling and feeding of the green peach aphid (Homoptera, Aphididae). Journal of Economic Entomology 86: 34-39

Rodriguez PA, Stam R, Warbroek T, Bos JIB (2014) Mp10 and Mp42 from the aphid species Myzus persicae trigger plant defenses in Nicotiana benthamiana through different activities. Molecular Plant-Microbe Interactions 27: 30-39

Schiavinato M, Strasser R, Mach L, Dohm JC, Himmelbauer H (2019) Genome and transcriptome characterization of the glycoengineered Nicotiana benthamiana line DeltaXT/FT. BMC Genomics 20: 594

\section{Schilmiller A, Shi F, Kim J, Charbonneau AL, Holmes D, Jones AD, Last RL (2010) Mass} spectrometry screening reveals widespread diversity in trichome specialized metabolites of tomato chromosomal substitution lines. Plant Journal 62: 391-403

Schilmiller AL, Charbonneau AL, Last RL (2012) Identification of a BAHD acetyltransferase that produces protective acyl sugars in tomato trichomes. Proc Natl Acad Sci U S A 109: $16377-16382$ 
Schilmiller AL, Moghe GD, Fan P, Ghosh B, Ning J, Jones AD, Last RL (2015) Functionally

1016

1017

1018

1019

1020

1021

1022

1023

1024

1025

1026

1027

1028

1029

1030

1031

1032

1033

1034

1035

1036

1037

1038

1039

1040

1041

1042

1043

1044 divergent alleles and duplicated Loci encoding an acyltransferase contribute to acylsugar metabolite diversity in Solanum trichomes. Plant Cell 27: 1002-1017

Schmittgen TD, Livak KJ (2008) Analyzing real-time PCR data by the comparative C(T) method. Nat Protoc 3: 1101-1108

Schneider CA, Rasband WS, Eliceiri KW (2012) NIH Image to ImageJ: 25 years of image analysis. Nature Methods 9: 671-675

Shepherd RW, Wagner GJ (2007) Phylloplane proteins: emerging defenses at the aerial frontline? Trends in Plant Science 12: 51-56

Simmons AT, Gurr GM, McGrath D, Martin PM, Nicol HI (2004) Entrapment of Helicoverpa armigera (Hubner) (Lepidoptera:Noctuidae) on glandular trichomes of Lycopersicon species. Australian Journal of Entomology 43: 196-200

Simon B, Cenis JL, Demichelis S, Rapisarda C, Caciagli P, Bosco D (2003) Survey of Bemisia tabaci (Hemiptera: Aleyrodidae) biotypes in Italy with the description of a new biotype (T) from Euphorbia characias. Bull Entomol Res 93: 259-264

Slocombe SP, Schauvinhold I, McQuinn RP, Besser K, Welsby NA, Harper A, Aziz N, Li Y, Larson TR, Giovannoni J, Dixon RA, Broun P (2008) Transcriptomic and reverse genetic analyses of branched-chain fatty acid and acyl sugar production in Solanum pennellii and Nicotiana benthamiana. Plant Physiol 148: 1830-1846

Song Z, Li S, Chen X, Liu L, Song Z (2006) Synthesis of sucrose esters. Forestry Studies in China 8: 26-29

Stamatakis A (2014) RAxML version 8: a tool for phylogenetic analysis and post-analysis of large phylogenies. Bioinformatics 30: 1312-1313

Stone WD, Pellicore MJ, Hagstrom S, Lawton TJ (2020) HSIviewer: Pushbutton hyperspectral image analysis for rapid plant phenotyping. in review

Thompson JD, Higgins DG, Gibson TJ (1994) Clustal-W - Improving the sensitivity of progressive multiple sequence alignment through sequence weighting, position-specific gap penalties and weight matrix choice. Nucleic Acids Research 22: 4673-4680

Thurston R (1961) Resistance in Nicotiana to the green peach aphids and some other tobacco insect pest. Journal of Economic Entomology 54: 946-949 
1045 Tzin V, Yang X, Jing X, Zhang K, Jander G, Douglas AE (2015) RNA interference against gut osmoregulatory genes in phloem-feeding insects. J Insect Physiol 79: 105-112

1047 Van Eck J, Keen P, Tjahjadi M (2019) Agrobacterium tumefaciens-mediated transformation of 1048 tomato. Methods Mol Biol 1864: 225-234

1049 Van T, Weinhold A, Ullah C, Dressel S, Schoettner M, Gase K, Gaquerel E, Xu SQ, Baldwin IT (2017) O-acyl sugars protect a wild tobacco from both native fungal pathogens and a specialist herbivore. Plant Physiology 174: 370-386

1052 Velásquez AC, Chakravarthy S, Martin GB (2009) Virus-induced gene silencing (VIGS) in Nicotiana benthamiana and tomato. J Vis Exp doi: 10.3791/1292

1054 Wagner GJ, Wang E, Shepherd RW (2004) New approaches for studying and exploiting an old protuberance, the plant trichome. Annals of Botany 93: 3-11

1056 Weinhold A, Baldwin IT (2011) Trichome-derived O-acyl sugars are a first meal for caterpillars that tags them for predation. Proceedings of the National Academy of Sciences of the United States of America 108: 7855-7859

Worrall EA, Bravo-Cazar A, Nilon AT, Fletcher SJ, Robinson KE, Carr JP, Mitter N (2019) Exogenous application of RNAii-inducing double-stranded rna inhibits aphidmediated transmission of a plant virus. Front Plant Sci 10: 265 

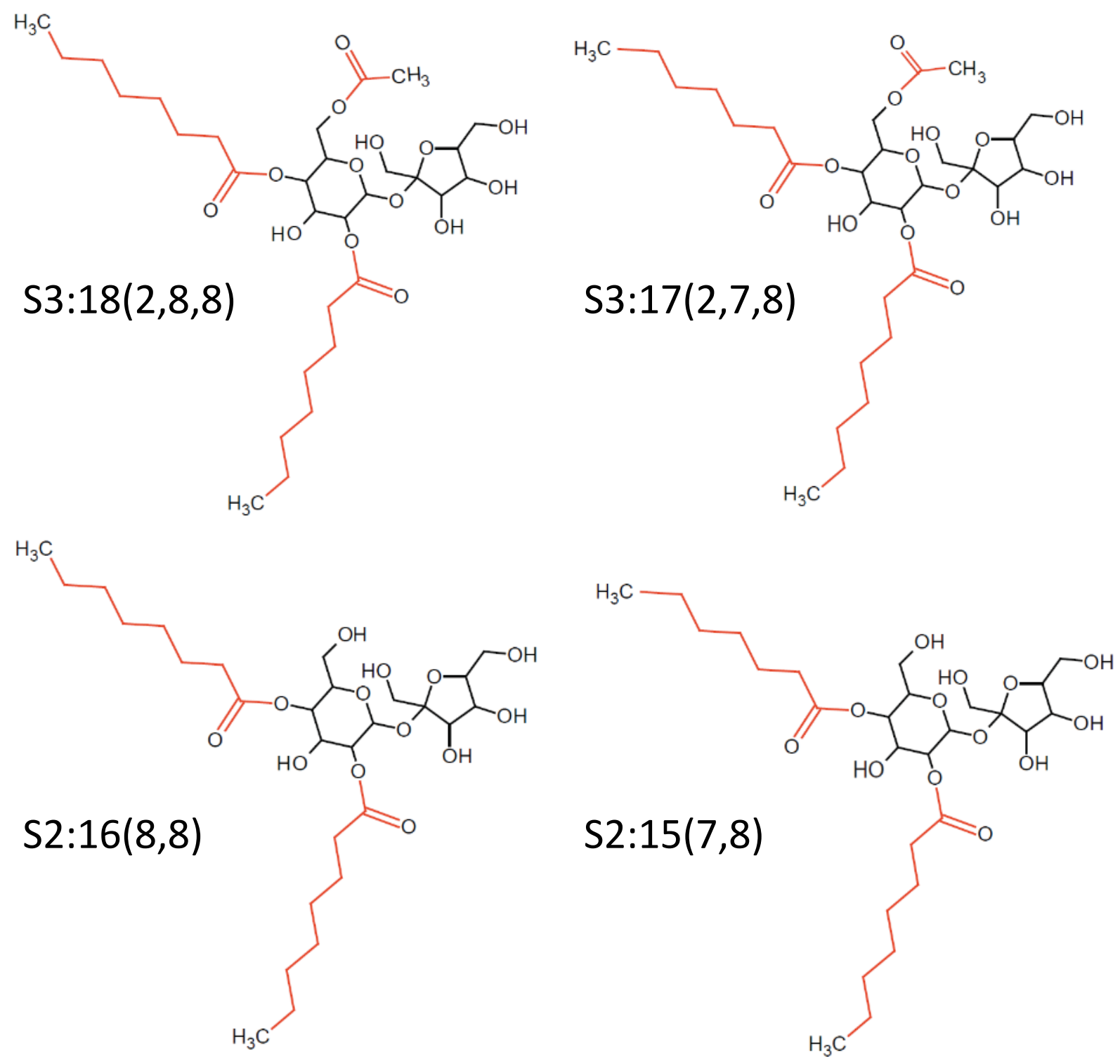

Figure 1. Predominant acylsugars in Nicotiana benthamiana

Acylsucroses S3:17(2,7,8), S3:18(2,8,8), S2:16(8,8) and S2:15(7,8) are present in $N$. benthamiana. In the acylsugar structure names, $\mathrm{S}$ refers to sucrose, followed by the number of acyl chains, the total length of acyl chains, and the length of each individual chain in parentheses. Although the presence of $\mathrm{C} 2, \mathrm{C} 7$, and $\mathrm{C} 8$ chain lengths is confirmed, the specific positions of the acyl chains on the sucrose molecule are hypothesized based on previous observations of acylsugars in Nicotiana alata (Moghe et al, 2017), the predicted evolution of the acylsugar biosynthetic pathway, and enzyme promiscuities in the Solanaceae family. 


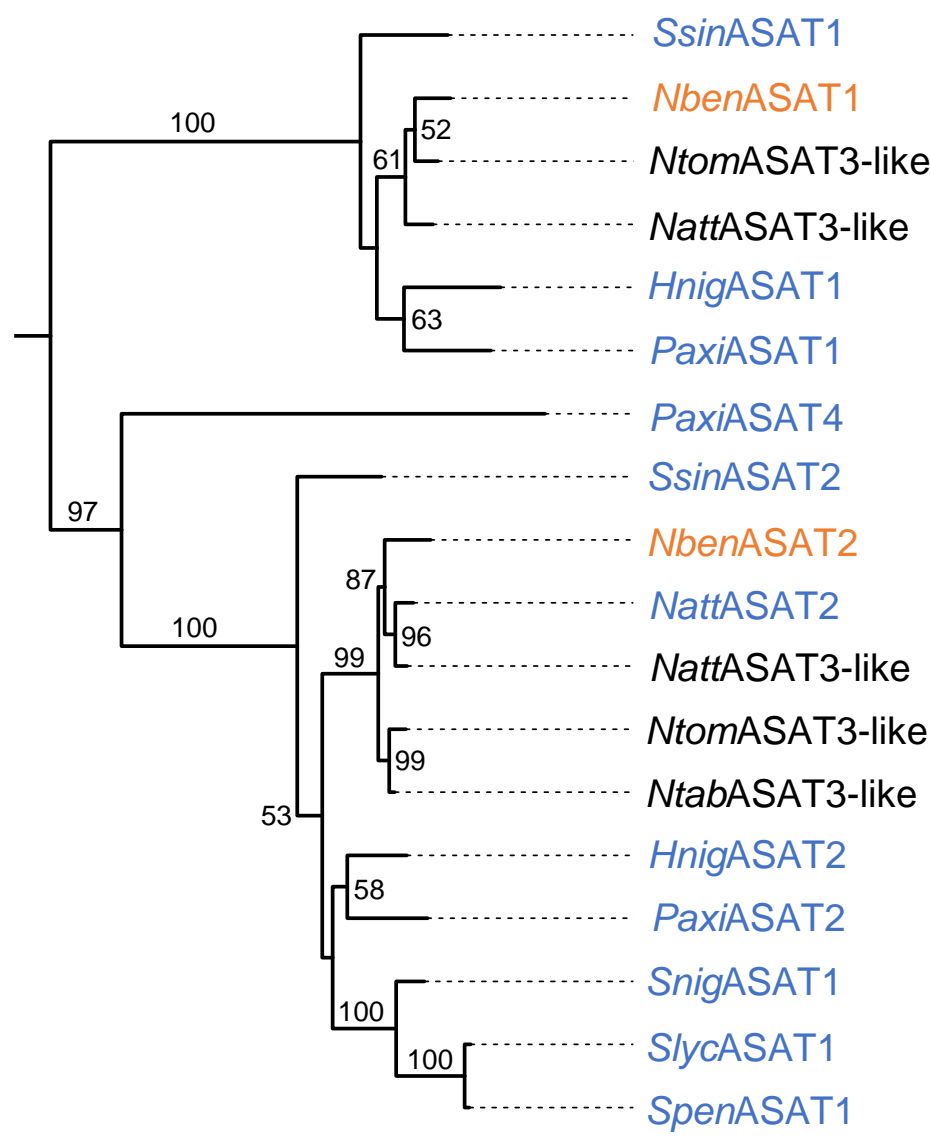

\section{Figure 2. ASAT phylogenetic tree}

The evolutionary history of ASATs in the Solanaceae (Tables S1 and S2) was inferred using the Maximum Likelihood method in RAxML. Presented is a subtree of a larger tree that includes all annotated ASATs (Figure S1). The branch labels indicate the percentage of trees in which the associated taxa clustered together (bootstrap of 1000). Only values greater than 50 are presented. The two predicted $N$. benthamiana ASATs are highlighted in orange and ASATs that were previously chemically characterized are highlighted in blue. 


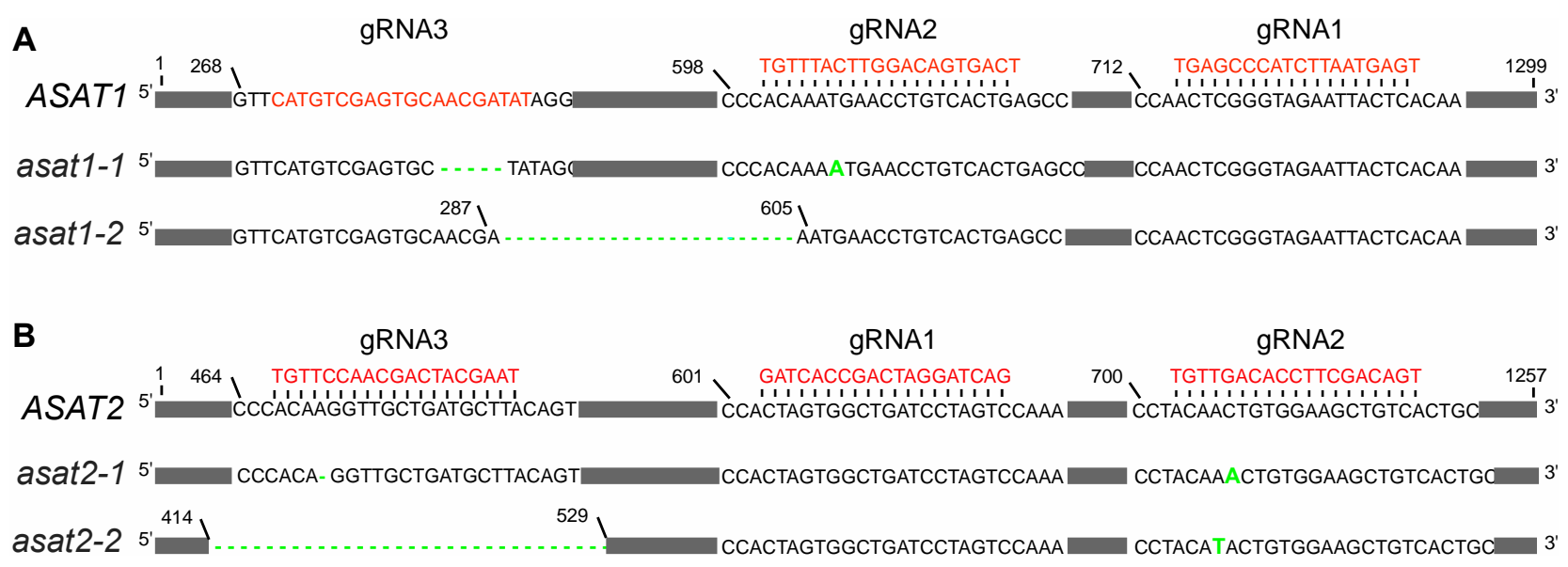

\section{Figure 3. $N$. benthamiana ASAT mutations produced with CRISPR/Cas9}

Three gRNAs (sequences shown in red) were designed to edit either ASAT1 or ASAT2.

Whereas gRNA3 for ASAT1 is on the sense strand, the other gRNAs are on the antisense strand. For both ASAT1 and ASAT2, we obtained two independent mutations resulting from the corresponding gRNA2 and gRNA3. Single-base mutations and deletions are shown in green. (A) asat 1-1 has a five-nucleotide deletion at gRNA3 and a single-nucleotide insertion at gRNA2. asat1-2 has a 318-nucleotide deletion between the gRNA3 and gRNA2 cutting sites. (B) asat2-1 has a single-nucleotide deletion at gRNA3 and single-nucleotide insertion at gRNA2 leading to a translation frame shift between the two mutations. asat2-2 has a 115nucleotide deletion at gRNA3 and a single-nucleotide insertion leading to a translation frame shift at gRNA2. 


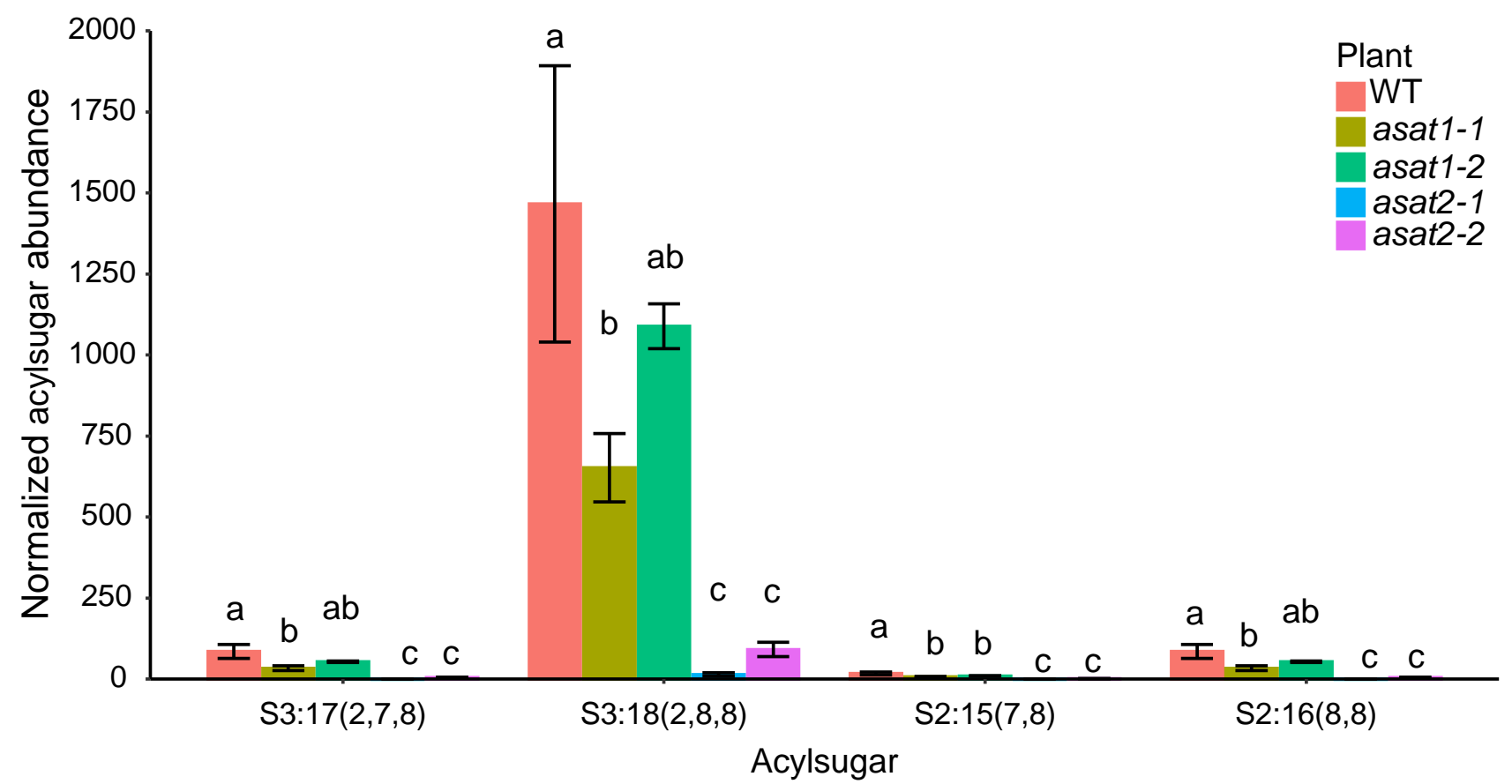

Figure 4. The abundances of two Nicotiana benthamiana acylsugars $(S 2: 17(2,7,8)$ and $S 2: 18(2,8,8))$, and two likely pathway intermediates/fragmentations $(\mathrm{S2:15}(7,8)$ and $S 2: 16(8,8))$

Acyl sugar LC/MS peak areas were normalized relative to the peak area of Telmisartan, which was added as an internal control, and then to the leaf dry weight (per gram). Error bars represent standard errors from measurements of three plants of each genotype. Significant differences for each acylsugar between different genotypes were tested using one-way ANOVA followed by a Duncan's post hoc test ( $p$ $<0.05)$. Differences between groups are denoted with letters. 
A Helicoverpa zea

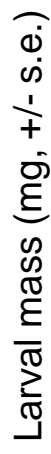

B Heliothis virescens

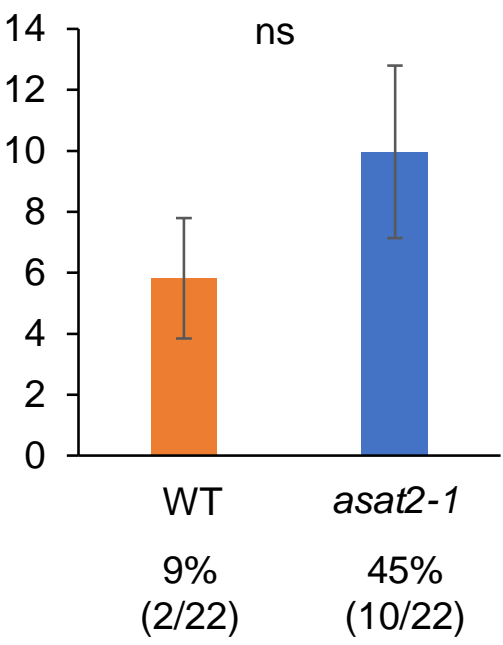

E Heliothis virescens

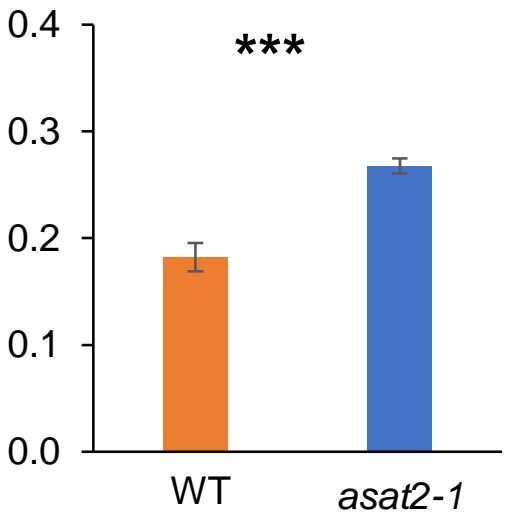

$94 \%$

$(15 / 16)$
C Trichoplusia ni

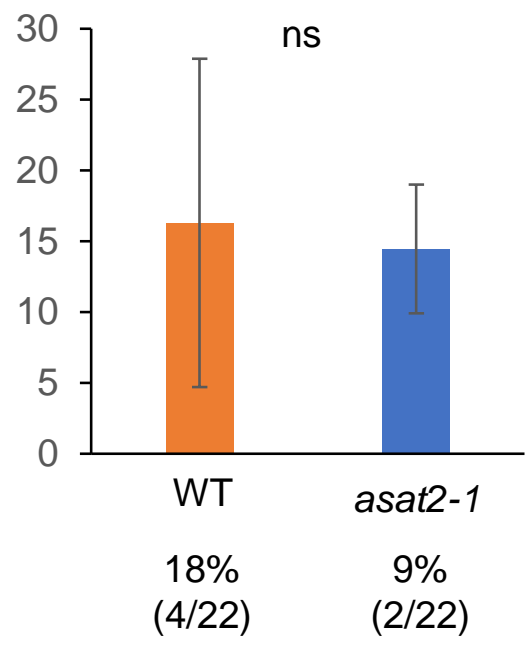

F Trichoplusia ni

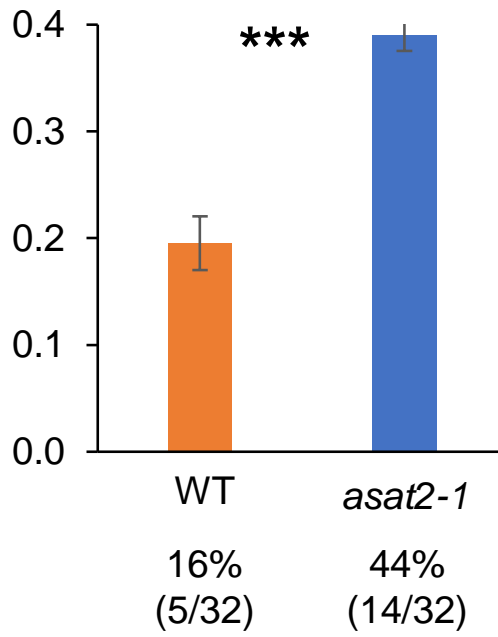

Figure 7. Caterpillar bioassays on wildtype (WT) and asat2-1 mutant Nicotiana benthamiana. $(\mathbf{A}, \mathbf{B}, \mathbf{C})$ Larval mass of surviving Helicoverpa zea, Heliothis virescens, and Trichoplusia ni after 10 days after being placed on plants as neonates. (D, E, F) Relative growth rate of surviving $H$. zea, $H$. virescens, and T. $n i$ on wildtype and nata2-1 plants. Insects were raised for five days on artificial diet, prior to 7 days of feeding on $N$. benthamiana. Percent survival (number of surviving insects/number of total insects) is shown below each figure. Mean $+/$ - s.e. ${ }^{* * *} p<0.001$, ns: no significant difference $(\mathrm{P}>0.05, t$-test $)$. 


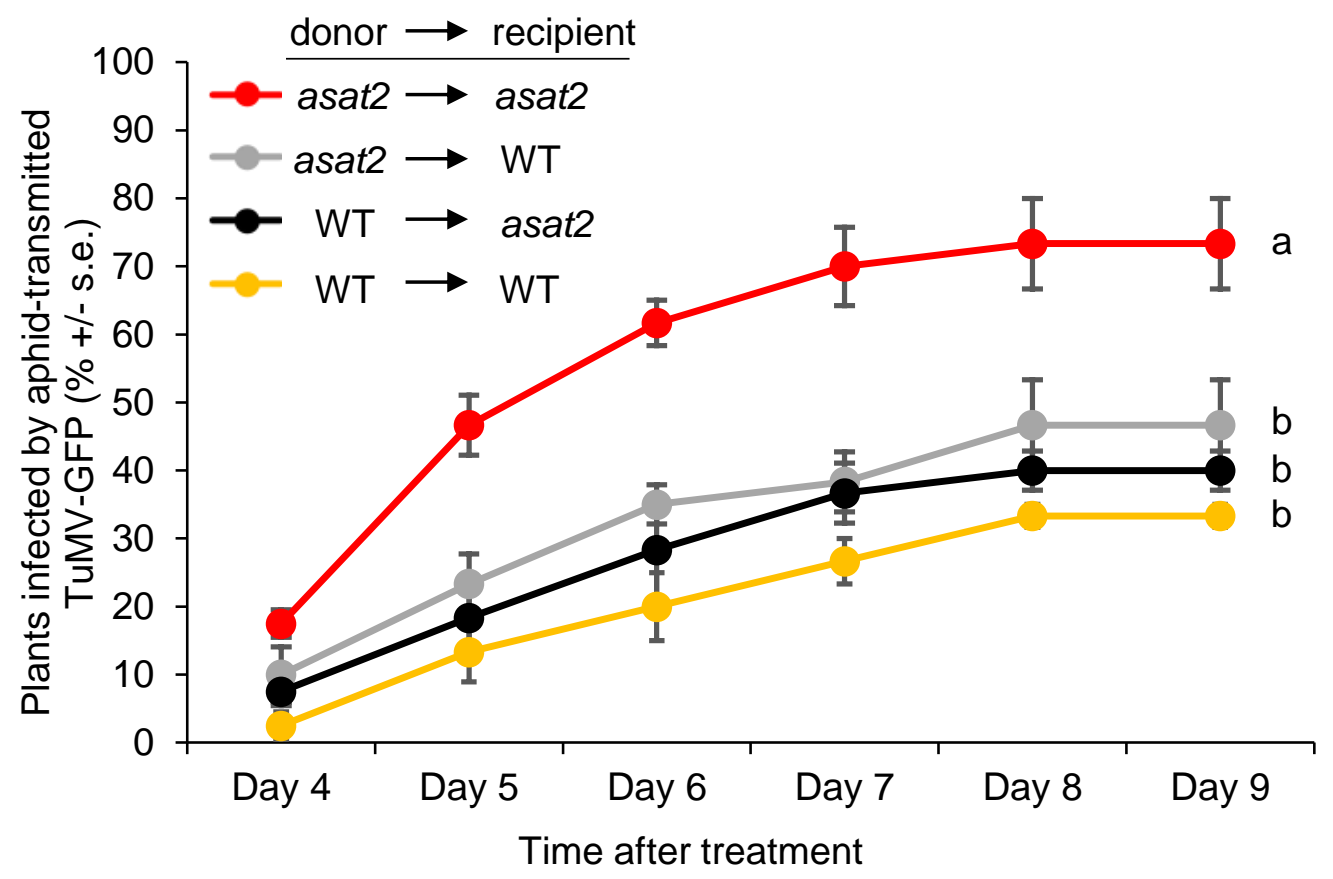

Figure 8. Aphid transmission of GFP-expressing turnip mosaic virus (TuMV-GFP) on wildtype (WT) and asat 2-1 plants. Aphids were transferred from $N$. benthamiana plants infected with TuMVGFP to uninfected plants and the percentage of GFP-expressing recipient plants was recorded over time. Data are mean $+/$ - s.e. of $N=60$, with data from three separate experiments combined. Statistical analyses were conducted using one-way ANOVA followed by a Tukey HSD post hoc test. Significant differences were observed from day 5 post treatment and thereafter. Lowercase letters denoted the differences between each group at day 9 post treatment $(p<0.05)$. WT: wildtype. 

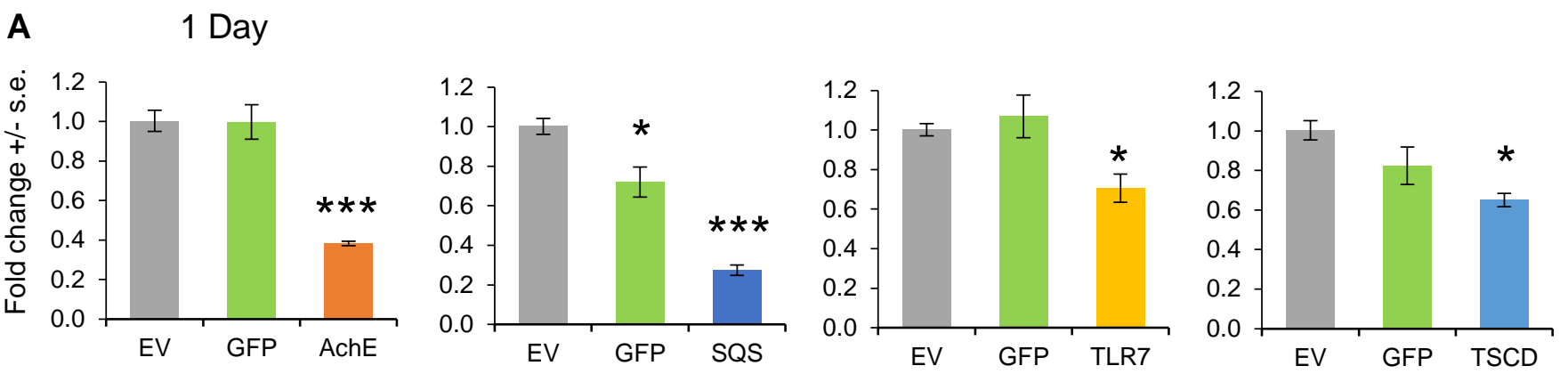

B

7 Days
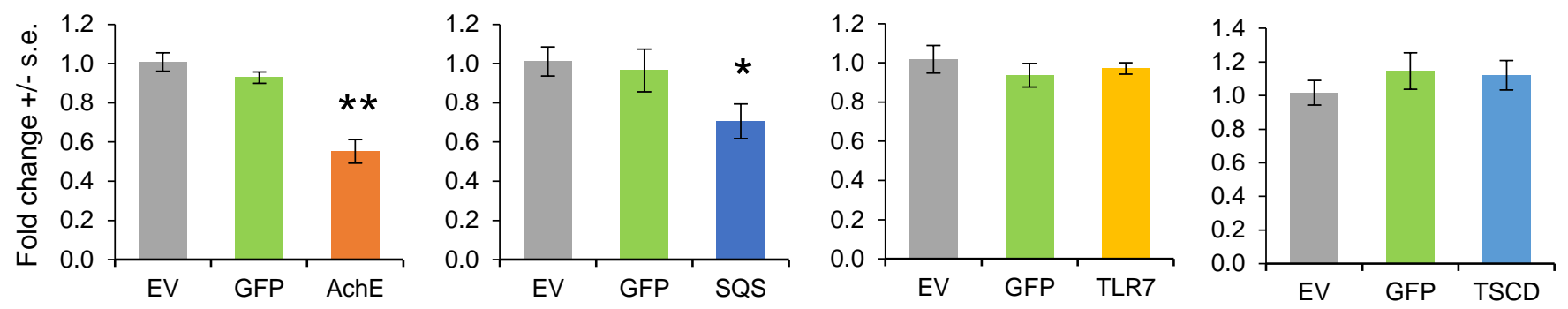

C Day 7 on asat2-1

D Day 7 on wildtype
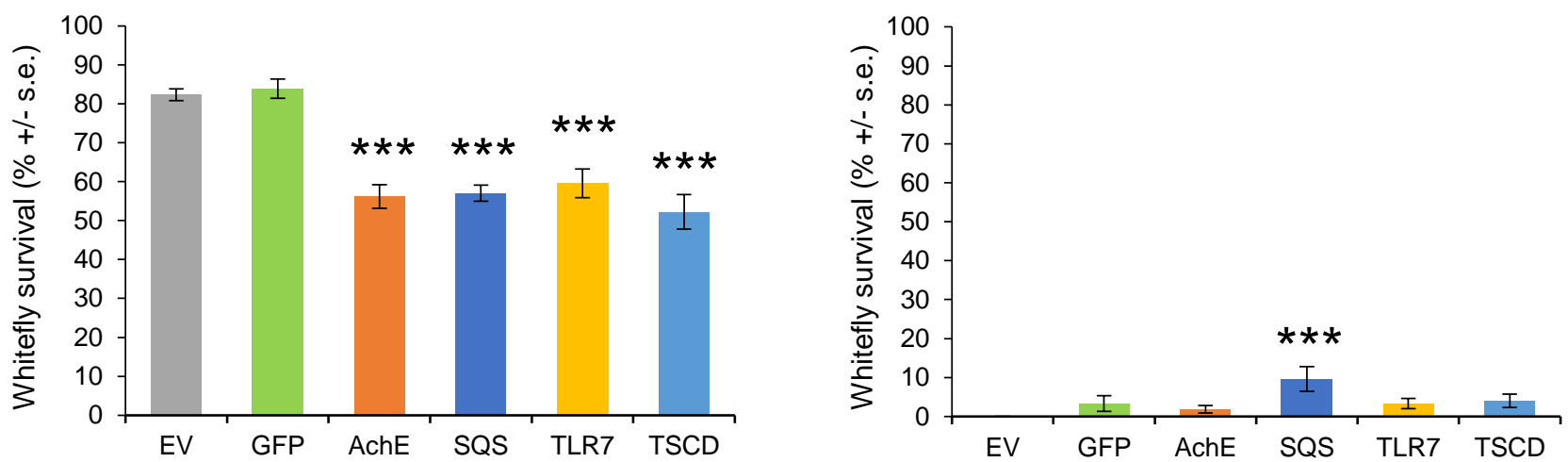

Figure 9. Virus induced gene silencing (VIGS) of whitefly genes using tobacco rattle virus. Whiteflies were placed on plants infected with TRV expressing VIGS constructs and gene expression was measured after (A) one and (B) seven days. In each experiment, the empty virus vector (EV) and GFP-carrying virus were used as controls. Gene expression was normalized to 1 for EV control plants. Whitefly survival was assessed on TRV-infected plants after (C) seven days of feeding on asat2-1 plants, and (D) seven days of feeding on wildtype plants. GFP/AchE/SQS/TLR7/TSCD: VIGS plants with TRV expressing RNA constructs targeting GFP, AchE, SQS, TLR7, and TSCD, respectively; AchE: acetylcholinesterase, SQS: squalene synthase, TLR7: toll-like receptor 7, TSCD: tubulin-specific chaperon D. The survival and the 7day qPCR experiments were done for three times. The qPCR experiments for 1-day were done twice. Significant differences were determined using one-way ANOVA with a fixed factor of treatments and a block effect of experiment, followed by a Dunnett's post hoc test for comparing VIGS constructs to the empty vector (EV) control. Mean $+/$ - s.e. of $\mathrm{n}=3(\mathrm{~A}), \mathrm{n}=9$ (B), and $\mathrm{n}=27(\mathrm{C}, \mathrm{D}){ }^{*} p<0.05 ; * * p<0.01$, $* * * p<0.001$. 

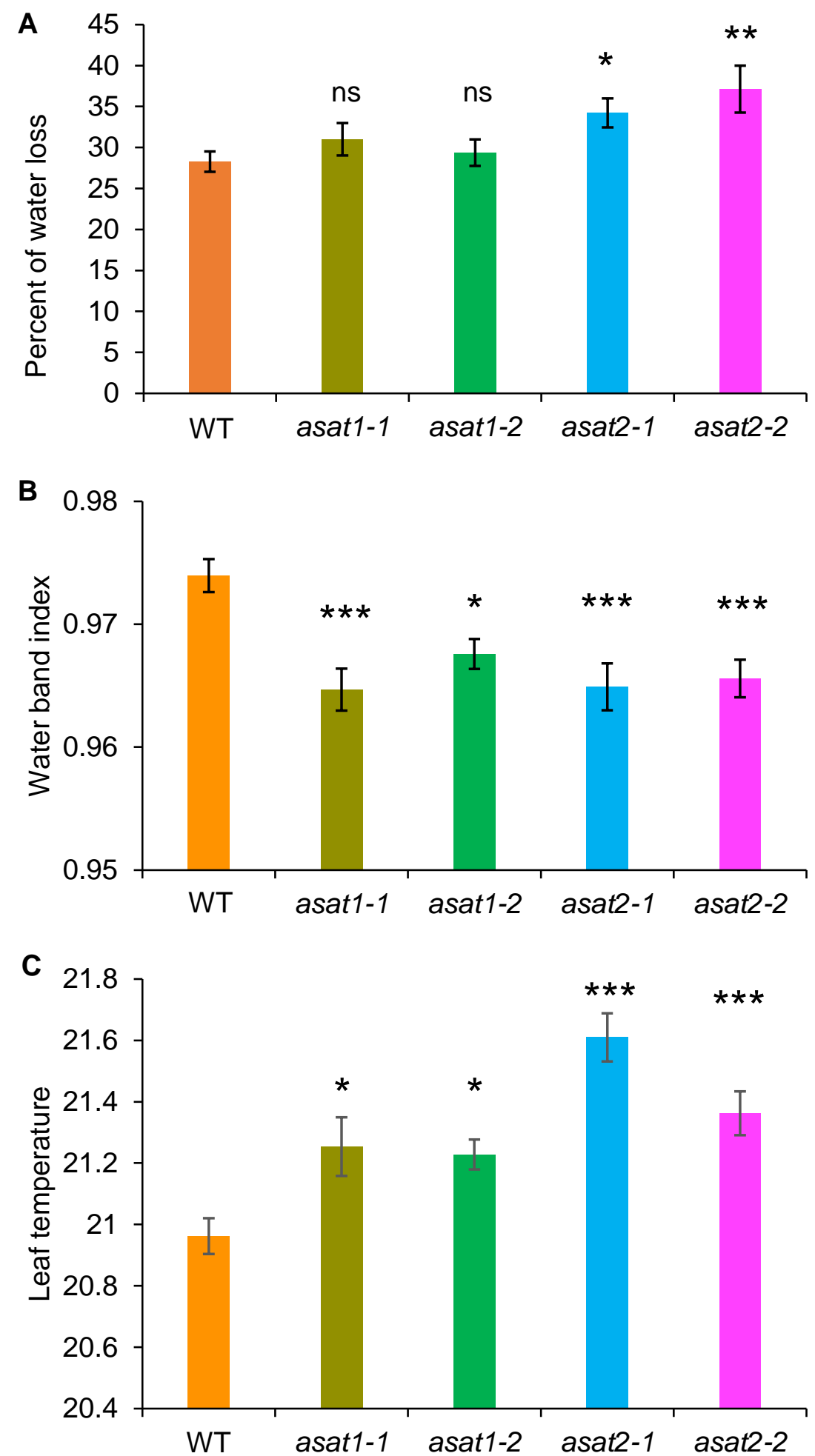

Figure 10. Water loss and leaf temperature of wildtype (WT), asat1, and asat2 Nicotiana benthamiana. (A) Percent of water loss from detached leaves in 24 hours, mean $+/$ - s.e. of $n=15$. (B) Leaf water content measured by the water band index from hyperspectral imaging, mean $+/-$ s.e. of $n=$ 20. (C) Leaf temperatures from leaves of different plant genotypes, mean $+/$ - s.e. of $n=20$. $n s=$ not significant, ${ }^{*} p<0.05, * * p<0.01, * * * p<0.001$, Dunnett's test relative to wildtype control. 


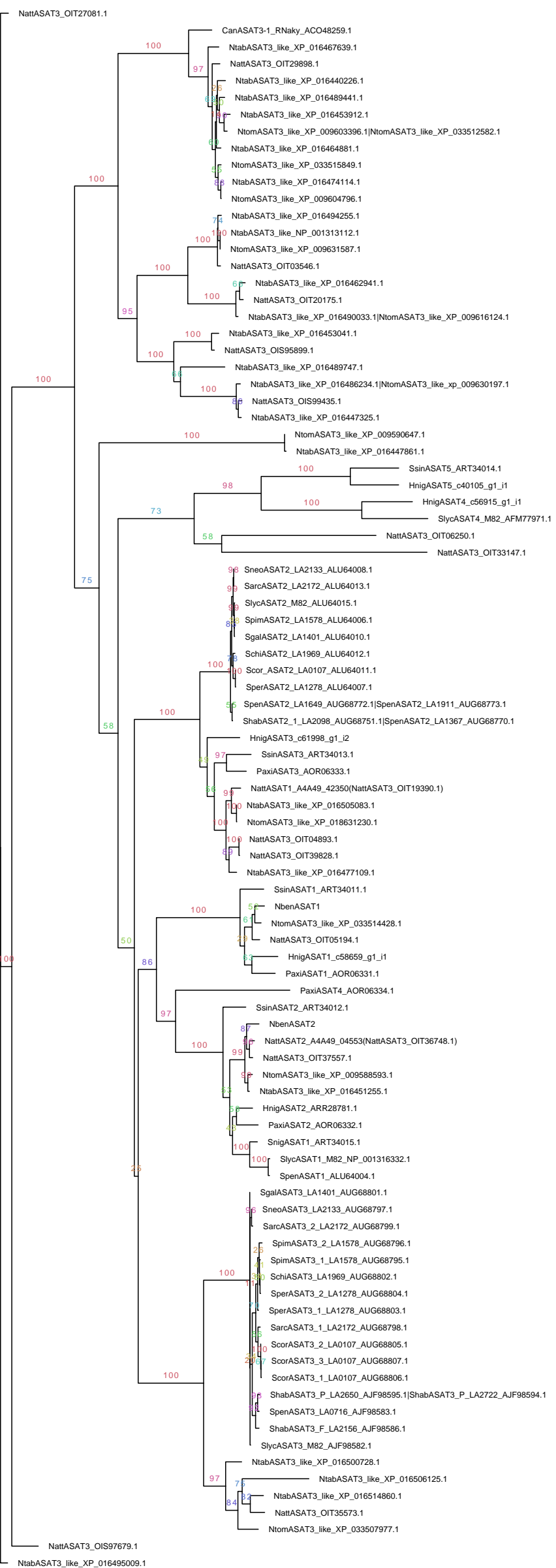

Figure S1. Phylogenetic analyses of annotated ASATs from Solanaceae species

The ASAT proteins used in constructing the tree are listed in Tables S1 and S2. The evolutionary history of ASATs from Solanaceae species was inferred by using the Maximum likelihood method in RAxML. The branch labels indicate the percentage of trees in which the associated taxa clustered together (bootstrap of 1000). 
HnASAT1 SSASAT1 NbASAT1 PaASAT1 PaASAT 4 SnASAT1 SPASAT1 SIASAT1 SSASAT2 NbASAT2 NaASAT2 HnASAT2 PaASAT2

101

HnASAT

SSASAT1 NbASAT1

PaASAT1

PaASAT 4

SnASAT1

SPASAT1

SIASAT1

SSASAT2

NbASAT2

NaASAT2

HnASAT2

PaASAT2

201

HnASAT1

SsASAT1

NbASAT1

PaASAT1

PaASAT 4

SnASAT1

SPASAT1

SIASAT1

SSASAT2

NbASAT2

NaASAT2

HAASAT2

PaASAT2

301

HnASAT1

SSASAT1

NbASAT1

PaASAT1

PaASAT 4

SnASAT1

SPASAT1

SIASAT1

SSASAT2

NbASAT2

NaASAT2

HnASAT2

PaASAT2

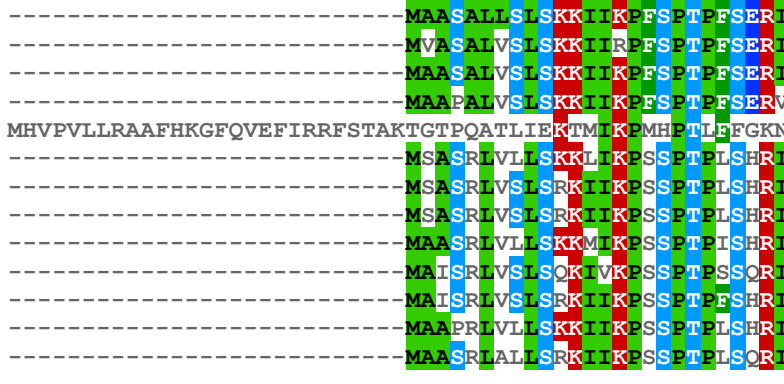

MVASALVSLSKKIIRPESPTPESERI YKLSE IDQFNSTQYIPLVFFYPKNKGN

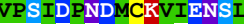

PSIDPNDMCKVIENSLS -IEPSDMCKVFENSLS SIEPSDMCKVIENSLS -IEPSDMCKVIENSLS YVPLVFFYPKNKG

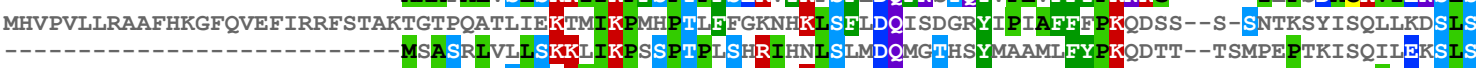
MSA SRL VSLSRKI IKPSSPTPLSHRI HKLSLMDQMGTHSYMAALIFYPKONTT--TSMPEPTKISQVLEKSLS MAASRLVLLSKKMIKPSSPTPISHRIHKLSLMDQMGTHSYVPFSFFYPKQDAA--S-SLEPTKVSRILEKSLS MAISRLVSLSRKIIKPSSPTPESHRIHKLSLMDQMGTHTYMPFSFFYPKQDTA--S-SLELTKVSQILEKSLS

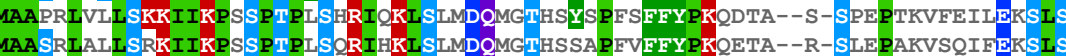

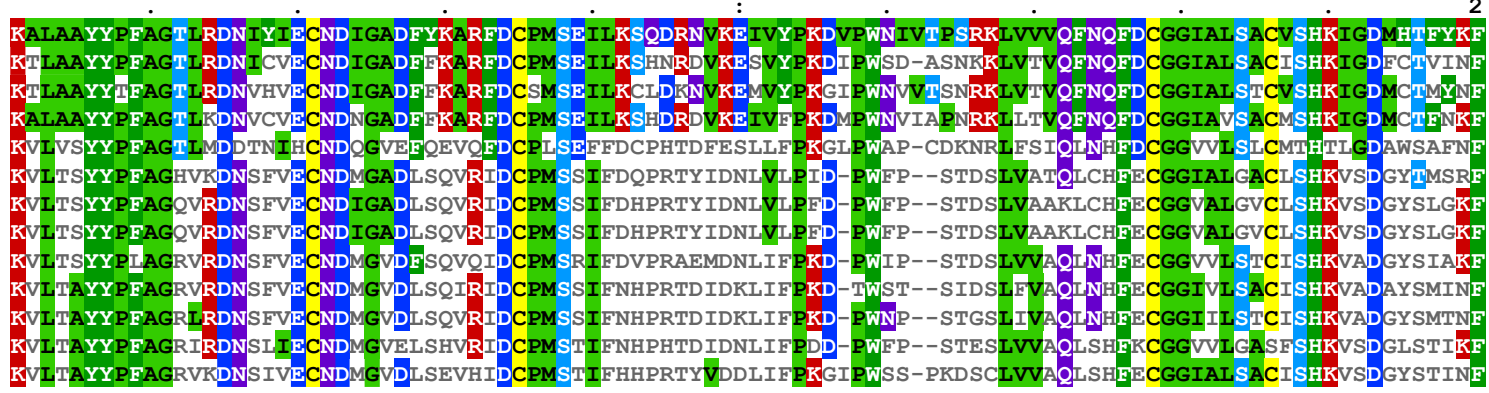

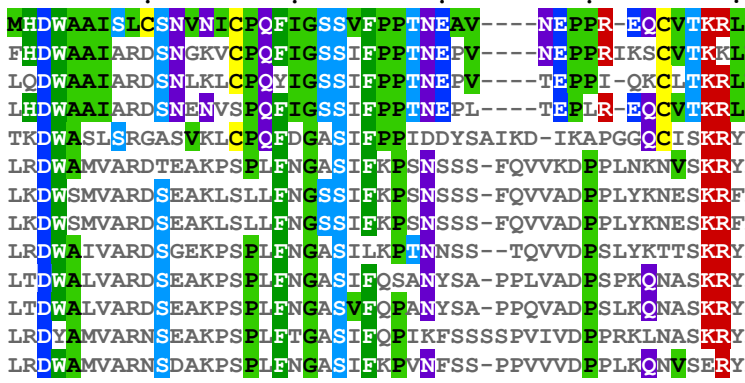

LRDWAMVARNSDAKPSPLENGASIFKPVNFSS-PPVVVDPPLKQNVSERYHESATKLKSLKALI--SADS--KSONIPTTVEAITAFLCKCVN-

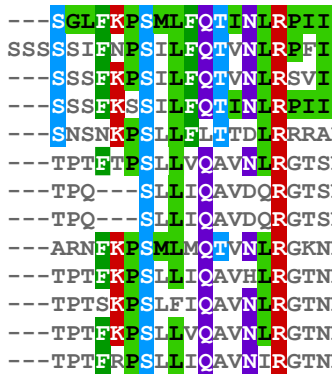

-PLPENTPGNFSSSLFVPTYTEEEMKLSRLVSELRKGKEKYFDDYRKCL

-PLPNNTA GNFSSS IFVPTYNEEEMKLSRLVSQLRKEKEQLVANYKKCKL -G

GGODLVSATKGPFOEIRKLYKEVDFO GDQDLVSTIIGPFQEIRKL FKDMDFD GDQDLVSTII I PEQEIRKLFKDMDFD GNTGVPLYIFAREKREMELQRLVGEFKKEKERVSSAYNNVT-RETLFPTTMETIGKAVSMLAEIDKLD

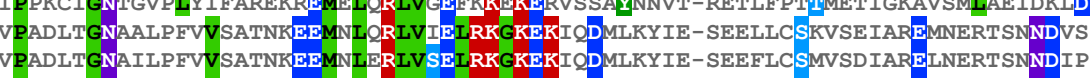

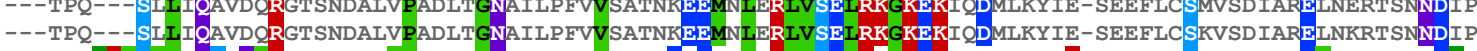
---ARNFKP SML MQTVNLRGKNNDALLPADLVGNVIFP FVVSAANED ENLQRLVSELREGKEKIHNTLECIK-SEELLC SKVCEIAREINKRTTRDDFS ---TPTFKPSLLIQAVHLRGTNNDALVPAGLVGNAVLPYLVSAANIEDLKLQRLIGELREGKEKVRHMLKDIK-SEDVLY SRVSELATQINERTSSNDFS ---TPTSKPSLF I QAVNLRGTNNDALVPAGLVGNA I LPYVASAANGEDLKWORL I GELRERKEKVRDMLKD IK-PEEVLS SRVSELATQINEGTSSNDES

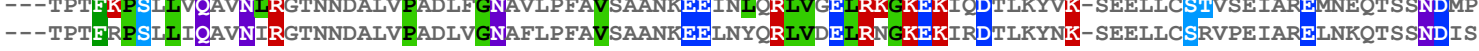

401

HnASAT 1 SSASAT1

NbASAT1

PaASAT1

PaASAT 4

SnASAT1

SPASAT1

SIASAT1

SSASAT2

NbASAT2

NaASAT2

HnASAT2

PaASAT2

LYRCSSLVNYGWHGLDFGWGMPNRVSMAD-VKLRNIFML FDNNTEDHVEAQV SFDKESKMSAFLREIFOVLEFKLHA

LYRCSSLANY PIYDVDFGWGRPKQITMAD-SPLRNTESLFDDNTGNYIEALVALDDEITMSTEEREMEQLIESOLPTEENKTEASAHC MYRCSSIANYPIYDIDFGWGKPNKISIAD-GVFRNVEIL YDNKI SDKLEAYVCLDEERTMSAFLKEMIEQFLEFEISSEEIKMEA--LFRCSSLVNY PLYDVDFGWGS PGKVIMAD-FLSRNFTTL LEDKTGEHLVAOVCEDNEITMSAEVREMEOLIEEELPSOEIYETQA-RC LYVSSSLSKLPAEDIDFGWGGPKRVIVHGGNYIQNVS ILCDNLRGD GIEVFVQLEE-QKMHALESAQ-EML EFASPYGGTIYRFTSIRKFPVNDMNEGWGRPRKVDV--ATYPINMFVLMDNQNGD GLEVIVNLDE-GKMSAEERND-ELIQIASPCSGLMYRFSSLRRFPSNDINEGWGRPRQVDF--STYPMNMEILMDNQNGDGVEVIASLQD-GELSALERND-EFLQ

MYRFSSIRRFPSNDINFGWGRPRQVDI--STFPINMEILMDNQNGDGVEVIASLQD-GELSALERND-DFLQFASPCLGF----.-MYRFTSVRKFPLNDIDFGWGRPRRADF--ATAPINLIFLMDDQSGDGVVLLNLGE-EEMSTEQSND-ELIQFASPS--

IYRFSSLRKLPFHDLNEGWGRPKIVDI--ATS PMNMFYLLENQNGDGVDVINLEE-GEMSLEESNE-ELLQEASLS-

IYRFSSLRKF P FHD INEGWGRPTRVDV--ATFPVNMFLLLDNQNGDGVEVVVNLEE-GEMSVEESNE-ELLQEASPSSGL

MYRFTSLRKFPL IDINEGWGRPRRVDM--ATYPVNMFVLMDNLSGDGVEVLVNLEE-GEMSAEESNN-ELHQEASPFSGL

IYRFTSLRKF P FHDINEGWGMPRKVDL--ATVPVNMEILIDNQSGGGLDVLVNLEQ-GEMSAEQSND-ELLHEASPSSGL

\section{Figure S2 Protein sequence alignment of $\mathrm{NbASAT1}$ and $\mathrm{NbASAT2}$ with other functionally} characterized ASATs in the Solanaceae species. The alignment was generated using Clustal Omega and visualized using Mview. Colors are coded based on the level of sequence identity. 
A

\begin{tabular}{clll}
\hline Parent ion $\mathbf{~} / \mathbf{z}$ & MS2 fragment/neutral loss peaks $\mathbf{~} / \mathbf{z}$ & t $^{\star}$ & Annotation \\
\hline 681.34 & $635.33,509.22,491.21,383.12,179.06,143.11,126.10,59.01,18.01$ & 1.76 & S3:18(2,8,8) Formate Adduct \\
671.30 & $681.34,671.30,635.33,509.22,143.11,126.10$ & 1.76 & S3:18(2,8,8) Chloride Adduct \\
635.32 & $509.22,491.21,467.21,383.12,365.11,179.06,143.11,126.10,18.01$ & 1.76 & S3:18(2,8,8) \\
639.32 & $593.32,467.21,449.20,323.10,143.11,126.10$ & 1.70 & S2:16(8,8) Intermediate Formate Adduct \\
593.32 & $467.21,323.10,143.11,126.10$ & 1.70 & S2:16(8,8) Intermediate \\
667.32 & $621.31,509.22,495.03,383.12,179.06,143.11,129.09,126.10,112.09$ & 1.70 & S3:17(2,7,8) Formate Adduct \\
621.31 & $495.03,383.12,365.11,143.11,129.09,126.10,59.01,18.01$ & 1.70 & S3:17(2,7,8) \\
625.31 & $579.30,467.21,453.20,323.10,143.11,129.09,126.10$ & 1.64 & S2:15(7,8) Intermediate Formate Adduct \\
555.23 & $509.22,383.12,143.11,126.10,59.01$ & 1.76 & S2:10(2,8) In-Source Fragment Formate Adduct \\
509.22 & $449.20,383.12,143.11,126.10,59.01$ & 1.76 & S2:10(2,8) In-Source Fragment \\
467.21 & $341.11,323.10,144.11,143.11$ & 1.70 & S1:8(8) In-Source Fragment \\
383.12 & $383.12,341.11,59.01$ & 1.76 & S1:2(2) In-Source Fragment \\
\hline
\end{tabular}

${ }^{*}$ rt: retention time of the predominant peak for each mass.

\section{B}

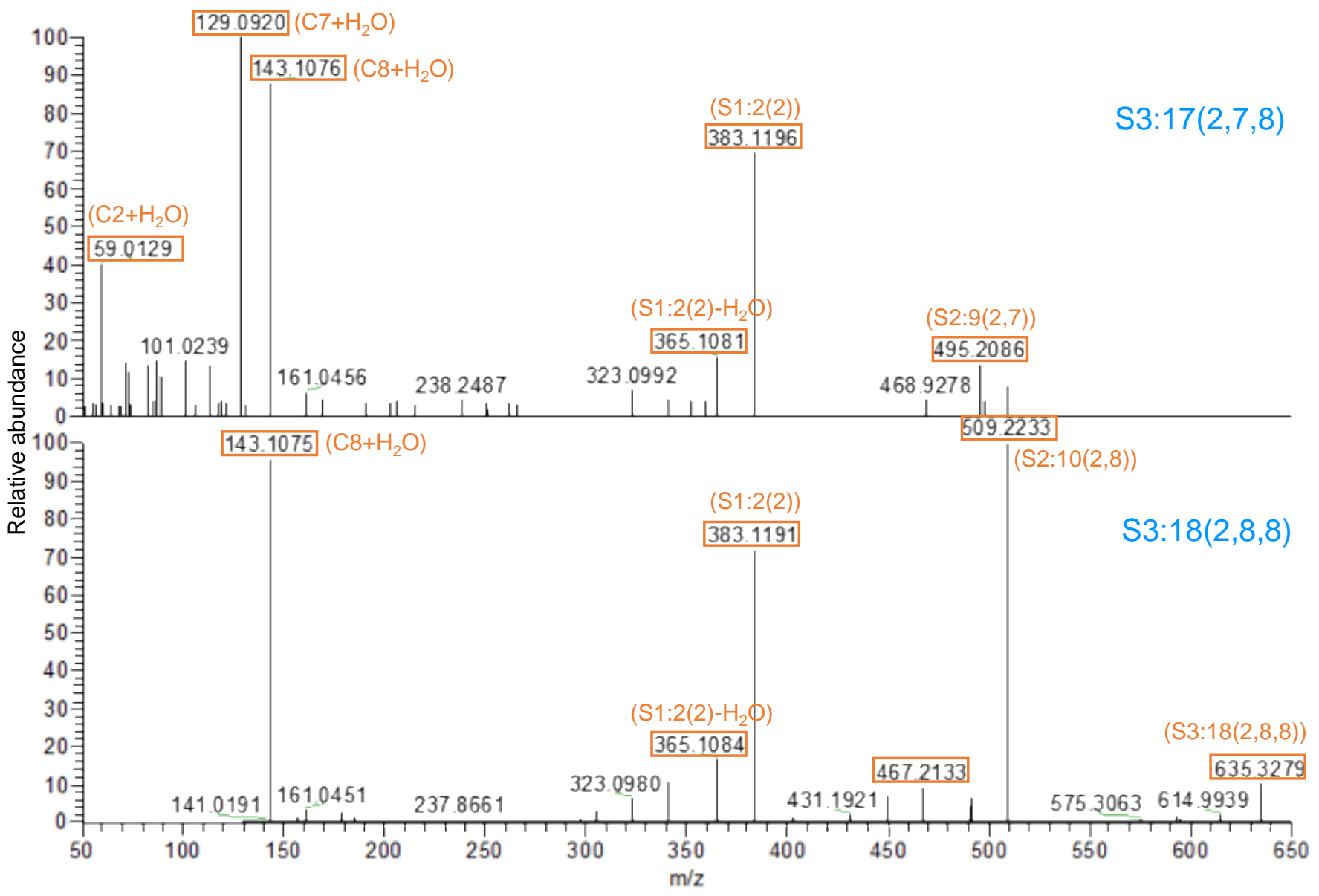

Figure S3. MS/MS chromatography of $N$. benthamiana acylsugars

(A) $N$. benthamiana acylsugar LC/MS peak identification and annotation (B) $\mathrm{MS}^{2}$ chromatography of the two predominant acyl sugars, S2:17(2,7,8) and S2:18 $(2,8,8)$. The names of the acylsugars are highlighted in blue and the chromatography is aligned on the $\mathrm{x}$ axis based on $\mathrm{m} / \mathrm{z}$. Highlighted in orange boxes are some of the abundant characteristic mass features, with their compositions in parenthesis. 

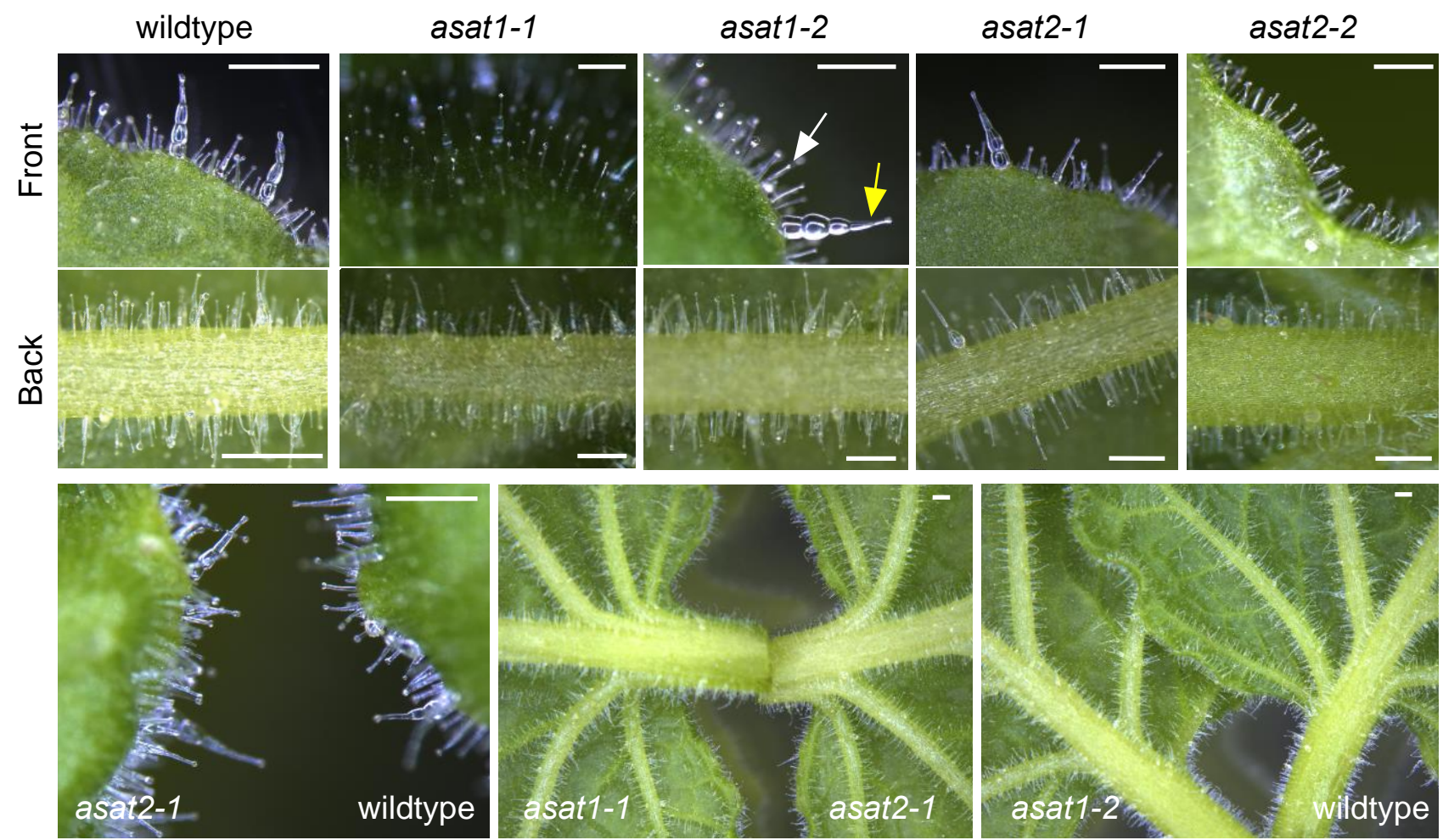

Figure S4. ASAT mutations do not affect trichome morphology and abundance

The white arrow indicates the small trichomes and yellow arrow indicates the large swollenstalk trichomes. Trichome pictures were taken from both the front and back sides of the leaf. Examples of leaves are put side-by-side from different genotypes to facilitate comparisons. Scale bars $=200 \mu \mathrm{m}$. 

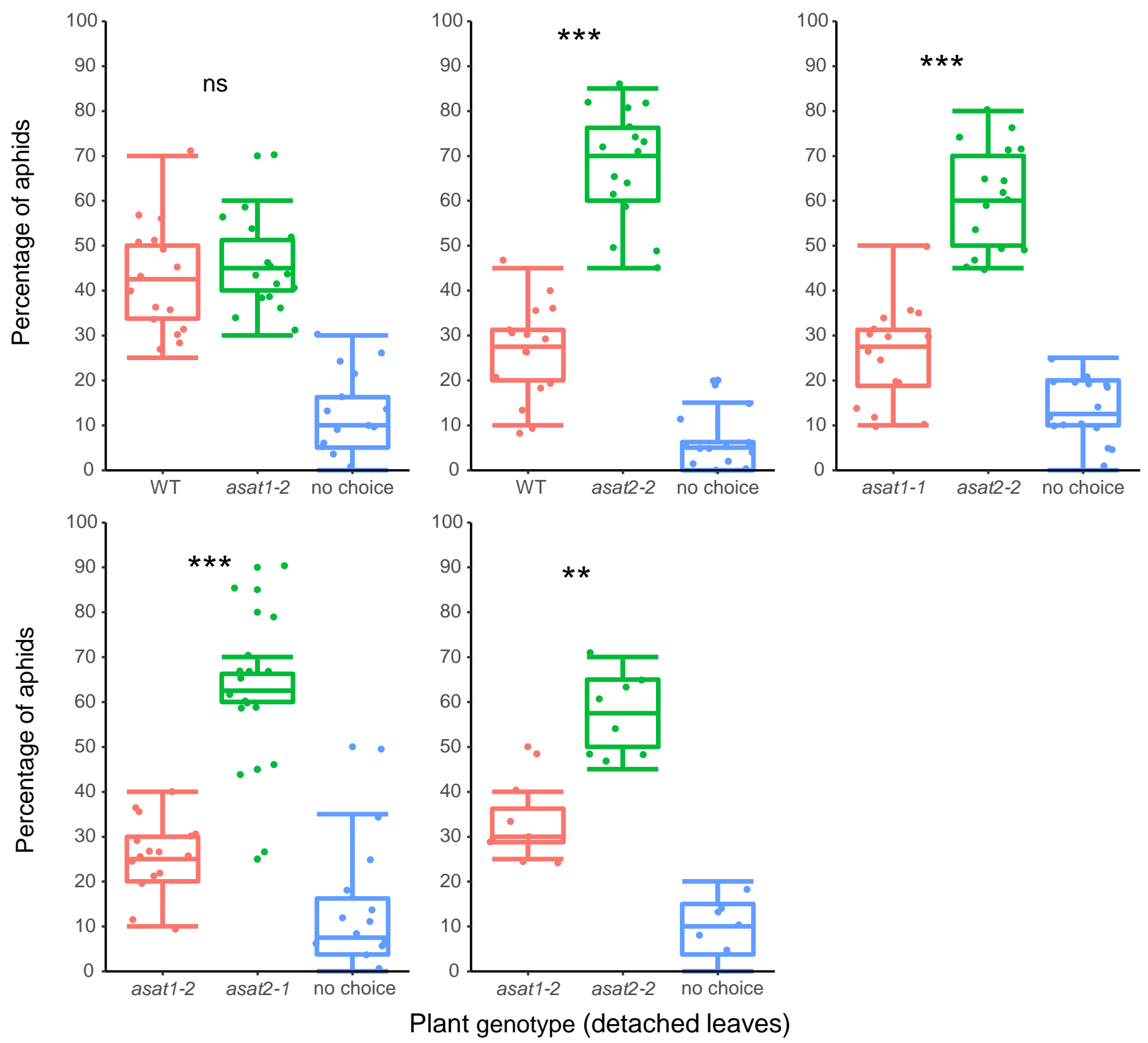

Figure S5. Pairwise aphid choice assay with wildtype and ASAT mutant leaves

Each experiment included detached leaves from 5-8 plants of each genotype and was repeated twice with similar results. Chi-square test was used for difference for all choice assays between plant genotypes. ${ }^{* *} p<0.01,{ }^{* *} p<0.001$, ns: not significant. no choice: aphids were elsewhere in the Petri dish and not on leaves at the end of the experiment. The box plots show the median, interquartile range, maximum and minimum after removal of outliers, and the individual data points. 


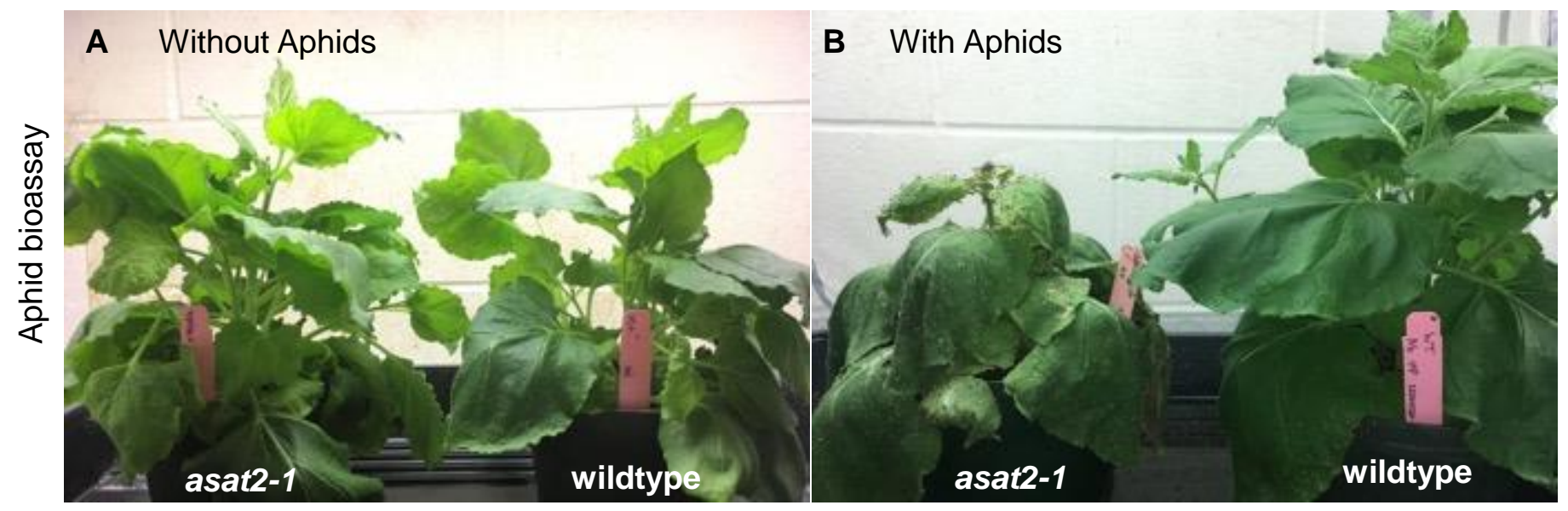

Figure S6. Aphid bioassays on wildtype and asat2-1 mutant $N$. benthamiana

(A) without $M$. persicae feeding. (B) with $M$. persicae feeding for one month. One wildtype (right) and one asat2-1 (left) plant were placed in the same tray in an insect cage. For the aphid challenge, ten adult M. persicae (from an aphid colony on N. tabacum) were released on each of the wildtype and the asat21 plants. The pictures were taken about one month after the aphid release. In the case of control plants without aphids (A), wildtype and asat2-1 look similar. (B) After one month of aphid feeding, the asat2-1 and wildtype plants look very different, with the wildtype plant looking vigorous and the asat2-1 line being smaller and wilted. On wildtype $N$. benthamiana, $M$. persicae reproduce slowly and sometimes not at all. If they do survive, they tend to feed on the undersides of senescing leaves. By contrast, $M$. persicae are able to establish a dense colony on the asat2-1 mutant and feed from the youngest leaves, which tend to be the most nutritious but also the best-defended. Plants in three replications of this experiment looked similar. 

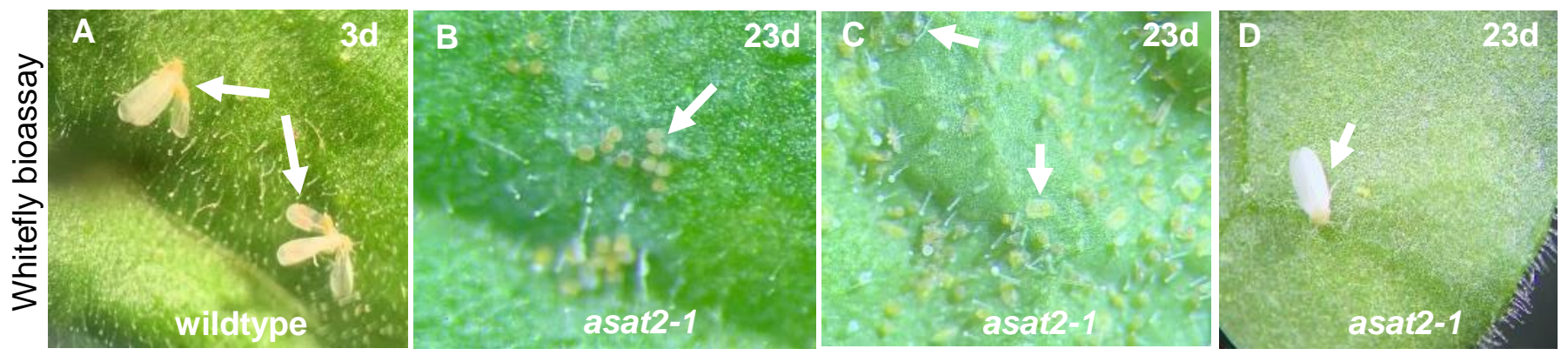

Figure S7. Whitefly bioassays on wildtype and asat2-1 mutant $N$. benthamiana

(A) Most whiteflies died within three days after release on wildtype $N$. benthamiana. Arrow indicates dead whiteflies. (B-D) Whiteflies survived and completed their life cycle on asat2-1 mutant $N$. benthamiana. At 23 days after release, whiteflies of different life stages were present on asat2-1 mutant plants. Arrows indicate eggs (B), eggshells and nymphs (C), and adults (D) on asat2-1 mutant plants. 


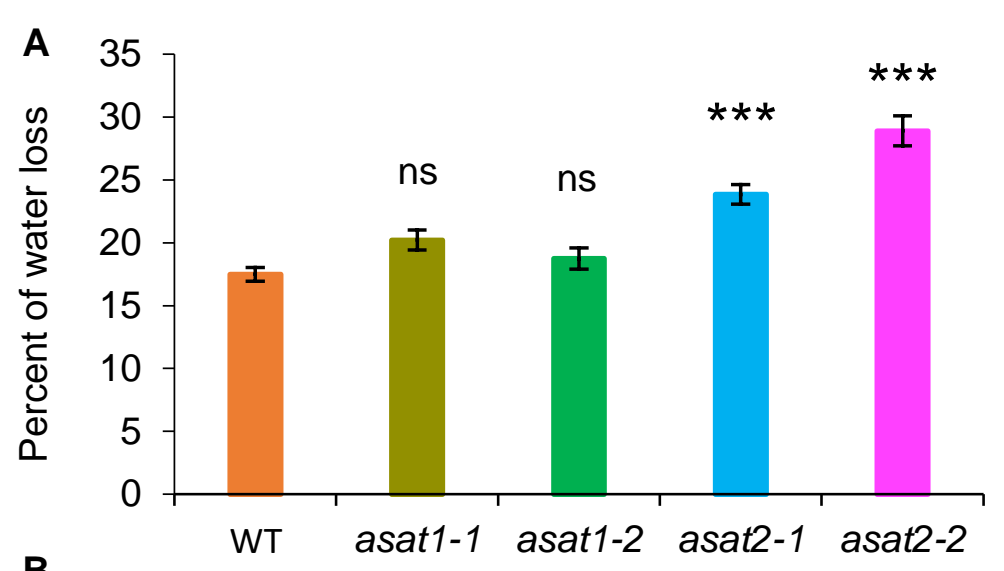

B
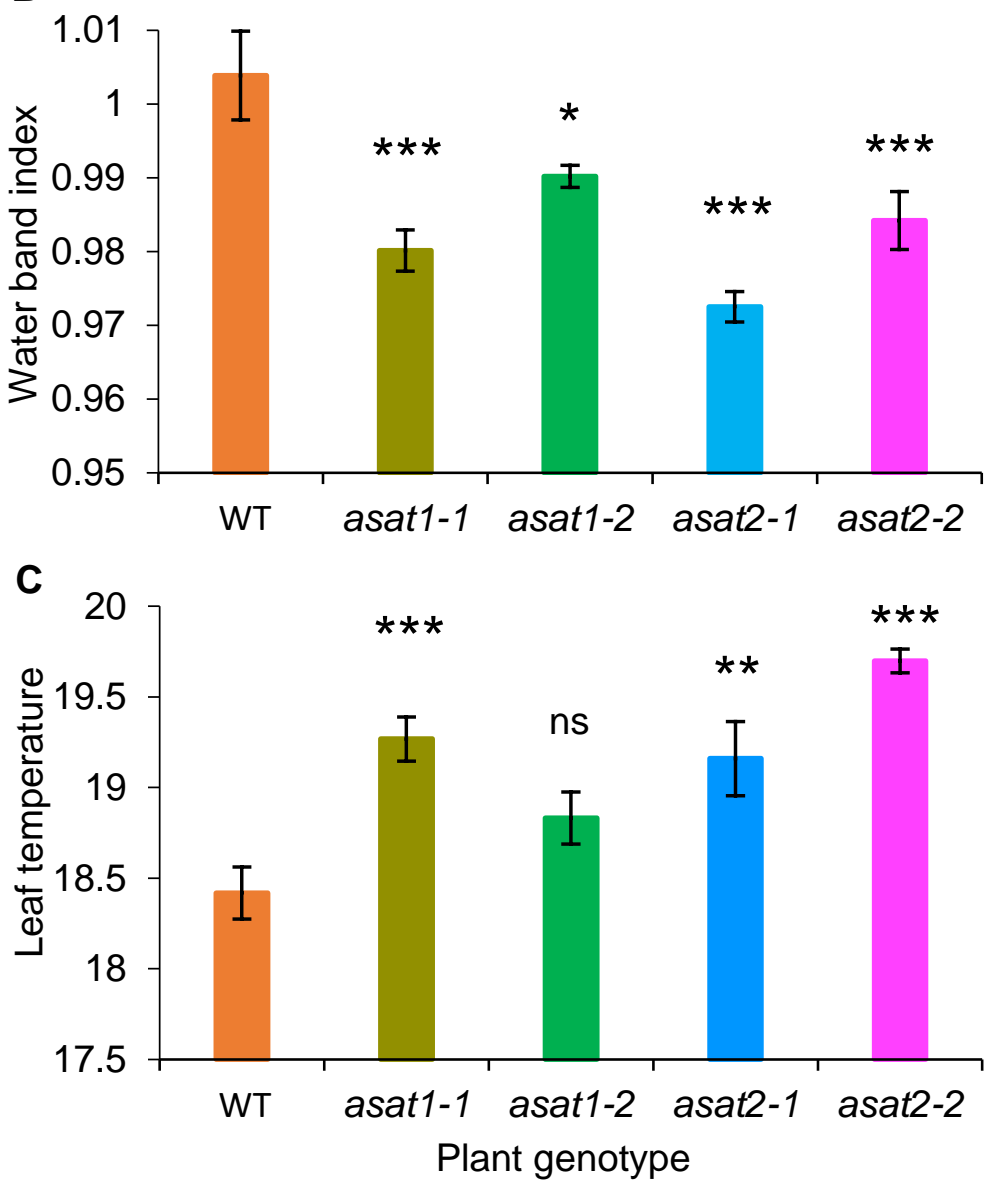

Figure S8. Independent repeat of water loss and leaf temperature shown in Figure 8.

(A) Percent of water loss from detached leaves after 24 hours, mean $+/$ - s.e. of $n=16$. (B) Leaf water content measured by the water band index from hyperspectral imaging, mean $+/$ - s.e. of $n=10$. (C) Leaf temperatures from leaves of different plant genotypes, mean $+/$ - s.e. of $n=10$ for wildtype and $n=5$ for mutants. ns, not significant, ${ }^{*} \mathrm{p}<0.05,{ }^{*} \mathrm{p}<0.01, * * * \mathrm{p}<0.001$, Dunnett's test relative to wildtype control. Error bars $=$ standard error. NS $=$ not significant. 


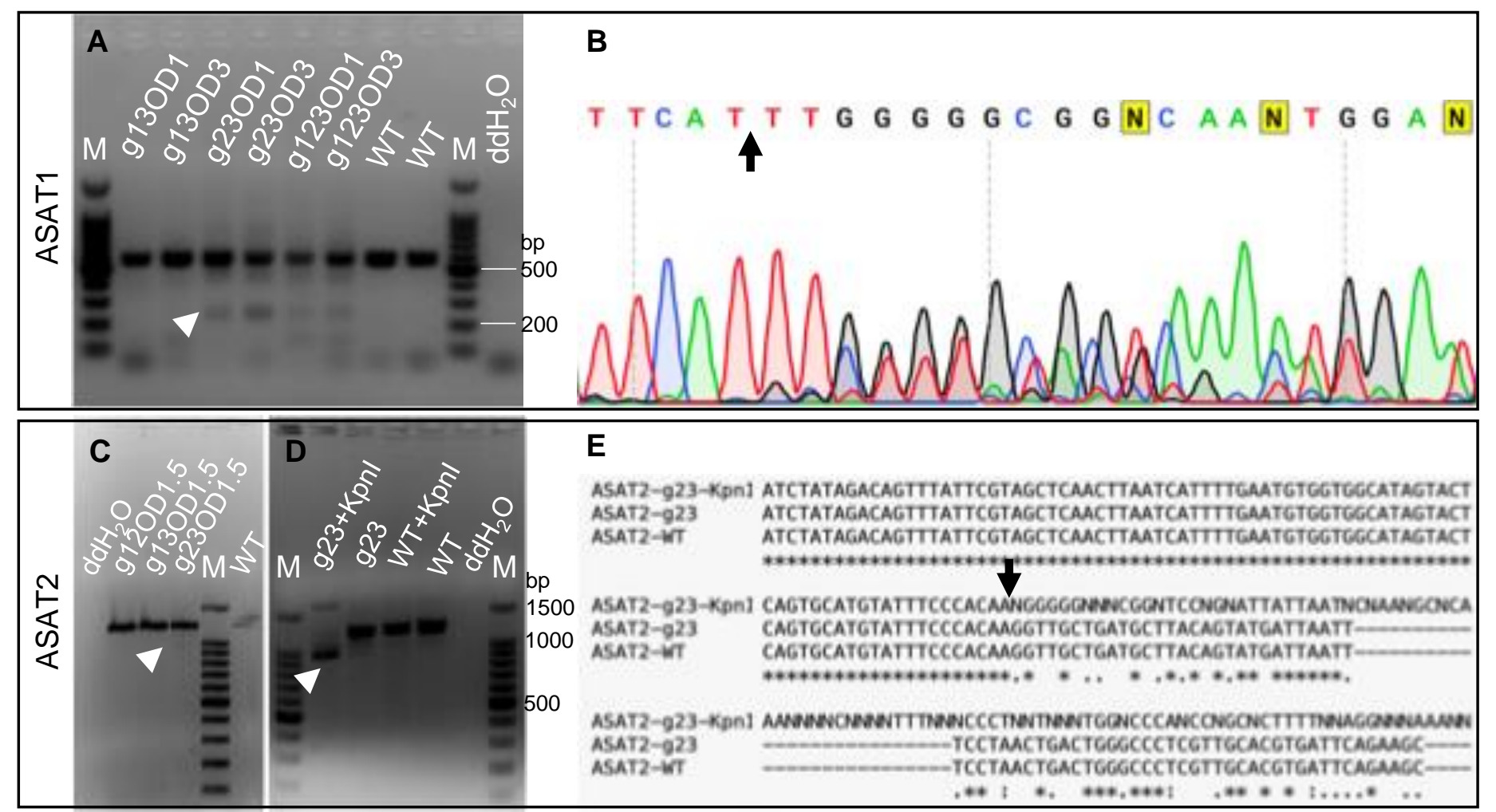

Figure S9. ASAT mutagenesis by transiently expressing Cas9 and gRNAs in $N$. benthamiana leaves via Agrobacterium infiltration

Three gRNAs each were used for ASAT1 and ASAT2 mutagenesis to test efficacy. (A) PCR of plants that were infiltrated with combinations of ASAT1 gRNA constructs. (B) DNA sequence confirmation of a representative sample (i.e. g23OD3) that displayed a shorter band in panel A. (C) PCR of plants that were infiltrated with combinations of ASAT2 gRNA constructs. (D) Restriction enzyme digestion site loss in PCR of g23OD1.5 (from panel C), where, prior to the PCR reaction, genomic DNA was digested with KpnI to remove wild type $A S A T 2$. The $K p n \mathrm{I}$ restriction site is only present in the expected deletion region. (E) Sequence confirmation of sample g23+KpnI from digestion site loss PCR in panel D. Arrow heads indicate the short versions of target genes after a deletion by the two working gRNA constructs. Arrows indicate the positions expected to be cut in gene sequences. The label for each lane in the gel: M, $100 \mathrm{bp}$ DNA marker ladder; WT, samples from wild type plants; ddH2O, no genomic DNA PCR control. 

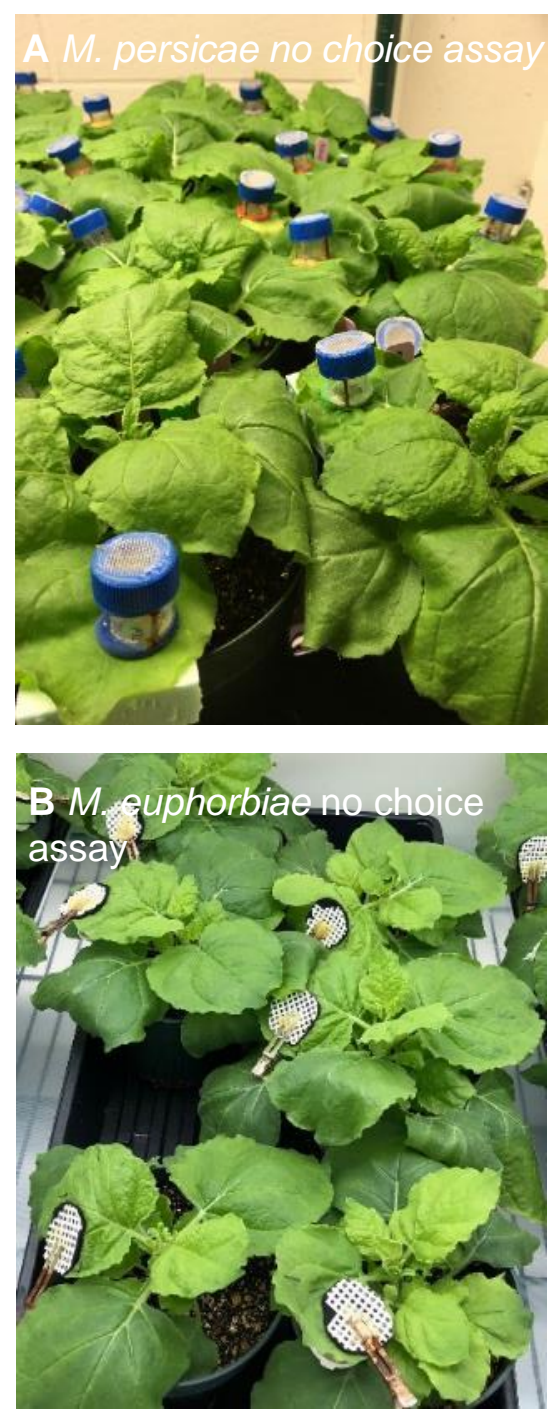

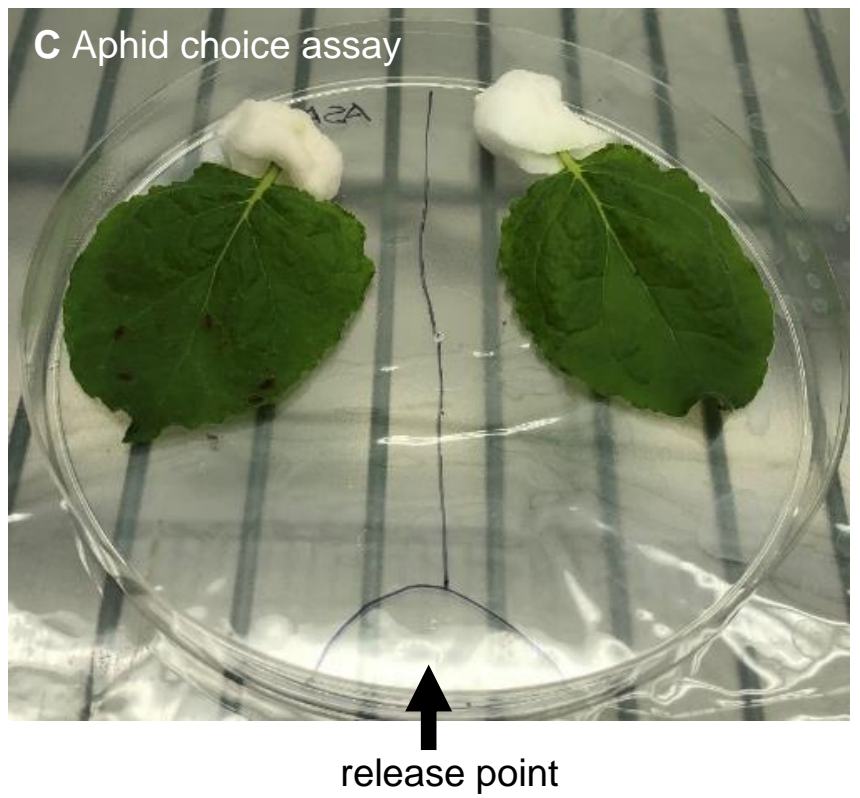

release point

Figure S10. Experimental setups for Myzus persicae bioassays

(A) Myzus persicae and (B) Macrosiphum euphorbiae no-choice assays. Aphids were caged on individual Nicotiana benthamiana leaves. Survival, reproduction, and size were assessed at time points described in the methods section. (C) Aphid choice assays. Two $N$. benthamiana leaves were placed in a $15 \mathrm{~cm}$ diameter Petri dish with their petioles inserted in moistened cotton swabs, ten aphids were released into the Petri dish at the indicated position, the dish was covered and placed under a 16:8 light:dark photoperiod, and, after 24 hours, the aphids residing on each leaf were counted. 